\title{
Uncertainty Across Volatility Regimes
}

\author{
Giovanni Angelini \\ University Ca' Foscari of Venice \\ Giovanni Caggiano \\ Emanuele Bacchiocchi \\ University of Milan \\ Luca Fanelli \\ Monash University and University of Padova* \\ University of Bologna
}

June 2018; Revised version: October 2018

\begin{abstract}
We propose a non-recursive identification scheme for uncertainty shocks which exploits breaks in the volatility of macroeconomic variables and is novel in the literature on uncertainty. This approach allows us to simultaneously address two major questions in the empirical literature: Is uncertainty a cause or effect of decline in economic activity? Does the relationship between uncertainty and economic activity change across macroeconomic regimes? Results based on a small-scale VAR with U.S. monthly data suggest that (i) uncertainty is an exogenous source of decline of economic activity, (ii) the effects of uncertainty shocks amplify in periods of economic and financial turmoil.
\end{abstract}

Keywords: Heteroskedasticity, Identification, Non-recursive SVAR, Uncertainty shocks, Volatility regime.

J.E.L.: C32, C51, E44, G01.

${ }^{*}$ Corresponding author: Department of Economics, 900 Dandenong Road, 3145 Caulfield East. Email: giovanni.caggiano@monash.edu. Telephone: +61 (0)3 99034516. 


\section{Introduction}

Since the aftermath of the recent Global Financial Crisis (GFC), there has been revamped attention on the role played by uncertainty as a driver of the business cycle. Three main findings have emerged from the extant literature: first, heightened uncertainty triggers a contraction in real activity; second, uncertainty tends to be higher during economic recessions; third, the effects of uncertainty shocks are not constant over time. The first finding is consistent with the theoretical literature that shows why uncertainty can have negative macroeconomic effects. The prevailing view is that uncertainty is recessionary in presence of real options effects (e.g. Bloom, 2009) or financial frictions (e.g. Christiano et al., 2014). However, uncertainty appears also to endogenously increase during recessions, as lower economic growth induces greater dispersion at the micro level and higher aggregate volatility. This second finding is consistent with the theoretical literature on 'endogenous uncertainty', which contends that uncertainty is rather a consequence, not a cause, of declining economic activity, as in e.g. Van Nieuwerburgh and Veldkamp (2006), Bachmann and Moscarini (2012), Fajgelbaum et al. (2017), Gourio (2014), Navarro (2014) and Plante et al. (2018). The fact that the relationship between uncertainty and real activity may not be constant over time is consistent with theoretical models that show how the effects of heightened uncertainty can be amplified in extreme conditions like high financial stress (e.g. Gilchrist et al., 2014; Alfaro et al., 2018; Arellano et al., 2018) or when monetary policy is constrained by the zero lower bound (Basu and Bundick, 2017).

Whether causality runs from uncertainty to real activity, or from real activity to uncertainty,

or in both directions, and whether this relationship changes under different macroeconomic conditions are issues which can be investigated empirically within a Structural VAR (SVAR) framework. The first issue requires moving away from recursive identification schemes, which are by construction ill suited to shed light on the reverse causality issue. This topic has been explicitly analyzed in Ludvigson et al. (2018a) and Carriero et al. (2018b), reporting mixed evidence. The second issue requires moving away from linear SVARs which would not allow to uncover possibly regime-dependent effects of uncertainty shocks. This concern has been addressed in the recent literature, and evidence that uncertainty shocks have time-varying effects has been provided by, among others, Alessandri and Mumtaz (2018), and Caggiano et al. (2014, 2017a). These early attempts of examining causality and time variation of uncertainty shocks have looked at the two issues in isolation. In light of the findings in the literature, however, this seems to be a strong limitation: if the relationship between uncertainty and real activity is indeed time-varying (or regime-dependent), it may very well be the case that also the direction of causality might change over time, something which a time-invariant SVAR would be unable to uncover. 
This paper fills this gap by proposing a non-recursively identified SVAR model which exploits breaks in the (unconditional) volatility of post-WW2 U.S. macroeconomic variables. Within this framework, we can allow both for on-impact effects of uncertainty on real activity, and vice versa, and for regime-dependence in these effects. As discussed in Magnusson and Mavroeidis (2014), structural breaks induced by policy shifts and/or the occurrence of financial crises, provide exogenous identifying information which can be fruitfully used for inference. The identification strategy we apply extends the standard 'identification-through-heteroskedasticity' approach, popularized in the empirical macroeconomic literature by Rigobon (2003), Rigobon and Sack (2003) and Lanne and Lütkepohl (2008), to the case where the structural parameters (on-impact coefficients), and hence the associated impulse response functions (IRFs), may vary across volatility regimes, see Bacchiocchi and Fanelli (2015) and Bacchiocchi et al. (2018). In this setup, changes in the VAR covariance matrix can be also ascribed to variations in the structural parameters and identification is achieved by imposing restrictions on the changes that characterize these parameters across volatility regimes. This opens up interesting possibilities for practitioners relative to 'standard' SVARs. In general, there are more moment conditions which can be used to identify the shocks jointly with theory-based restrictions, and the method is flexible enough to jointly allow for recursive and non-recursive structures across volatility regimes, provided a necessary and sufficient rank condition is respected. This is particularly important when addressing the issue of exogeneity/endogeneity of uncertainty, since it endows us with a formal test for exogeneity with the highly desirable property of accounting for potential dependence to macroeconomic (volatility) regimes.

We estimate, as in Ludvigson et al. (2018a), a small-scale SVAR with three variables: a measure of real activity, $Y_{t}$; an index of macroeconomic uncertainty, $U_{M t}$; and an index of financial uncertainty, $U_{F t}$. Real activity is proxied by either industrial production or employment, and the indices of macroeconomic and financial uncertainty are taken from Jurado et al. (2015) and Ludvigson et al. (2018a), respectively $!^{1}$ As argued in Ludvigson et al. (2018a), the joint use of macroeconomic and financial uncertainty indices is crucial to correctly uncover the relationship between uncertainty and real activity, since they can display substantially different properties. Data are monthly, and span the 1960-2015 sample. Using recursive and rolling-windows estimates of the VAR covariance matrix, we show that two main volatility breaks are consistent with the pattern of data, and can be associated with two important episodes of the U.S. history: one is the onset of the Great Moderation, and the other is the GFC of 2007-2008. This leads to the

\footnotetext{
${ }^{1}$ Other measures of macro uncertainty available in the literature have been proposed by Rossi et al. (2016) and Scotti (2016). We use the measure proposed by Jurado et al. (2015) to be consistent with the VAR specification in Ludvigson et al. (2018a), see below. In Carriero et al. (2018a) uncertainty and its effects are instead estimated in a single step within the same model.
} 
identification of three broad volatility regimes in the data, which correspond to three well-known macroeconomic regimes: the 'Great Inflation' period (1960M8-1984M3), the 'Great Moderation' period (1984M4-2007M12) and the 'Great Recession+Slow Recovery' period (2008M1-2015M4) 2 We then identify shocks by specifying a non-recursive structural model which exploits the differences in the average level of volatility displayed by macroeconomic variables in the three different sub-samples.

Our main findings can be summarized as follows. First, macroeconomic uncertainty can be better described as an exogenous driver of the U.S. business cycle. Macroeconomic uncertainty shocks trigger a decline of U.S. real economic activity, whose magnitude and persistence is estimated to be larger during Great Recession+Slow Recovery period, while the opposite is not supported by the empirical evidence. This finding holds true in all three macroeconomic regimes and is robust to several perturbations of the baseline model, such as the use of alternative measures of real activity and macroeconomic uncertainty, and also controlling for financial stress. Second, from the Great Moderation onwards, the pass-through of financial uncertainty to real economic activity is found to be indirect: financial uncertainty shocks trigger macroeconomic uncertainty and, via this channel, a contraction in real activity, with effects which amplify after the GFC. Financial uncertainty does not respond to real economic activity shocks nor to macroeconomic uncertainty shocks. Third, the estimated impulse responses differ substantially from those coming from a benchmark represented by a SVAR identified with heteroskedasticity along the lines of Lanne and Lütkepohl's (2008) method, i.e. by imposing that the autoregressive and the structural parameters are fixed across volatility regimes.

Overall, our findings support the claim that uncertainty, both macro and financial, is an exogenous driver of the business cycle, with contractionary effects on real activity that change over time. While we share the exogeneity of financial uncertainty with other contributions (e.g., Ludvigson et al., 2018a), one key finding of our paper is the exogeneity of macroeconomic uncertainty. We explicitly test this assumption in our structural model, and do not reject it. To this end, we consider two overidentified non-recursive SVARs, one featuring 'endogenous' macroeconomic uncertainty in the three volatility regimes, and a restricted (nested) version in which macroeconomic uncertainty does not respond contemporaneously to real activity shocks in the three volatility regimes. The SVAR with 'endogenous' macroeconomic uncertainty (and exogenous financial uncertainty) is rejected at the $5 \%$ significant level, while the SVAR featuring 'exogenous' macroeconomic uncertainty (and exogenous financial uncertainty) is supported by the data. Both specifications implicitly assume that financial uncertainty does not respond on

\footnotetext{
${ }^{2}$ Given the strong and well established association between the (average) volatility of most macroeconomic variables and specific macroeconomic regimes of U.S. economic history (e.g. McConnel and Perez-Quiros, 2000), throughout the paper we use the terms 'volatility regime' and 'macroeconomic regime' interchangeably.
} 
impact to negative economic shocks. It is important to stress that this assumption, which is required to jointly identify economic activity and macro uncertainty shocks, is not arbitrary but is supported by the reduced form evidence associated with the estimated SVAR, which suggests that financial uncertainty is poorly correlated with real economic activity until the beginning of the Great Recession+Slow Recovery period, and is correlated with macroeconomic uncertainty only starting from the beginning of the 1980s.

The closest papers to ours are Ludvigson et al. (2018a) and Carriero et al (2018b). Both papers deal with the issue of exogeneity/endogeneity of uncertainty. Similarly to Ludvigson et al. (2018a), our results are consistent with the view that financial uncertainty is exogenous to the business cycle. However, in stark contrast with their findings and in line with Carriero et al. (2018b), we find strong evidence that macroeconomic uncertainty is an exogenous driver of the business cycle $3^{3}$

Ludvigson et al. (2018a) propose a novel set-identification strategy in a time-invariant framework, that allows the joint identification of uncertainty and real activity shocks, without imposing any restrictions on the contemporaneous relations (see also Ludvigson et al., 2018b). Their identification strategy uses two types of shock-based restrictions. The first is what they label 'event constraints', which require that the identified financial uncertainty shocks must be large enough during two major financial disruptions, e.g. the 1987 stock market crash and the 2007-09 financial crisis. The second set of constraints are 'correlation constraints', which require that (i) macroeconomic and financial uncertainty shocks must be negatively correlated with aggregate stock market returns, and (ii) financial uncertainty shocks must be more highly correlated with stock market returns than macroeconomic uncertainty shocks. Using the same VAR specification as ours, they find that only financial uncertainty can be considered exogenous to the business cycle, while macroeconomic uncertainty should be treated as an endogenous response to business cycle fluctuations. They also find that while financial uncertainty shocks are contractionary shocks, macro uncertainty shocks have positive effects on real activity, in line with 'growth-options' theories. This major difference on the role of macroeconomic uncertainty can be explained by considering the different identification methods. In Ludvigson et al. (2018a), identification is based on external information, which is used asymmetrically between the two types of shocks: event constraints are imposed only on financial uncertainty shocks, and it is therefore unclear what is the actual identification information behind macroeconomic uncertainty. Moreover, relative to their analysis, the flexibility of our SVAR allows us to uncover relevant regime-dependent effects: financial uncertainty becomes a crucial factor for business

\footnotetext{
${ }^{3}$ To save space, a more comprehensive discussion of how our paper is connected to the large empirical literature on the identification of uncertainty shocks can be found in the Technical Supplement.
} 
cycle developments only after the 1980s. This result lines up with Ng and Wright (2013)'s argument that financial factors have played a crucial role in driving the U.S. business cycle after the mid 1980s, and is consistent with Caldara et al. (2016) and Caldara and Scotti (2018). Interestingly, our non-recursive SVAR shows that financial uncertainty affects real economic activity mostly indirectly, by fostering greater macroeconomic uncertainty.

Carriero et al. (2018b) jointly identify real activity and uncertainty shocks by using a novel stochastic volatility approach in the context of bivariate VARs which feature measures of macroeconomic and financial uncertainty (one at a time), along with measures of real economic activity. Accordingly, they do not separately identify the effects of macroeconomic and financial sources of uncertainty on economic fluctuations. Their empirical evidence is partly consistent with ours: they also document that macroeconomic uncertainty is broadly exogenous to business cycle fluctuations, while they find that financial uncertainty might, at least in part, arise as an endogenous response to some macroeconomic developments. The identification approach in Carriero et al. (2018b) is based on a stochastic volatility mechanism, hence it is inherently different from our heteroskedasticity-based approach to identification. Our method requires the occurrence of separate variance regimes which must be either known or inferred from the data, and this may possibly affect the inference and identification results if the volatility breaks are misspecified. The stochastic volatility approach in Carriero et al. (2018b) hinges on the specification of an independent stochastic process which governs the changes of the variances over time. This adds flexibility to the model and facilitates identification issues, but also raises computational issues. For instance, the extension of Carriero et al. (2018b)'s approach to the case of three-variate SVARs, which would allow to separately identify the effects of macroeconomic and financial uncertainty shocks, may become computationally demanding. Moreover, our approach allows, without imposing, regime-specific effects of uncertainty shocks, which may uncover important changes in the transmission mechanism over time, as we find for the effects of financial uncertainty.

The paper is organized as follows. Section 2 introduces the identification problem and presents our non-recursive identification approach. Section 3 discusses the data and the empirical results obtained from the estimated SVAR. Section 4 provides some concluding remarks. Additional technical details and empirical results and robustness checks are confined in an on-line Technical Supplement $4^{4}$

\footnotetext{
${ }^{4}$ Available online at https://drive.google.com/file/d/1cK3HPPWPEc7fG7J_VNafNalDYy0n66_g/view
} 


\section{Econometric framework}

In this Section, we outline our econometric methodology to deal with both regime-dependence and the joint identification of uncertainty and real activity shocks. Subsection 2.1 presents the general setup and discusses the nature of the problem one faces in 'standard' SVARs, while Subsection 2.2 extends the analysis to the 'identification-through-heteroskedasticity' method exploited in the paper.

\subsection{Identifying uncertainty and real economic activity shocks under homoskedas- ticity}

Consider the following SVAR:

$$
X_{t}=c+\Phi_{1} X_{t-1}+\ldots+\Phi_{p} X_{t-p}+B e_{t}=\Pi W_{t}+B e_{t} \quad, \quad e_{t} \sim \mathrm{WN}\left(0_{n \times 1}, I_{n}\right), \quad t=1, \ldots, T
$$

where $T$ is the sample length, $p$ is the system lag order, $X_{t}$ is the $n \times 1$ vector of endogenous variables, $c$ is a $n \times 1$ constant, $\Phi_{i}, i=1, \ldots, p$ are $n \times n$ matrices of parameters, $\Pi:=\left(\Phi_{1}, \ldots, \Phi_{p}, c\right)$, $W_{t}:=\left(X_{t-1}^{\prime}, \ldots X_{t-p}^{\prime}, 1\right)^{\prime}, B$ is a $n \times n$ non-singular matrix containing what we call 'structural parameters', and $e_{t}$ is the vector of mean zero, (normalized) unit variance and uncorrelated structural shocks. It is assumed that the autoregressive polynomial $\Phi(L):=I_{n}-\Phi_{1} L-\ldots-\Phi_{p} L^{p}$ is such that the solutions to $\operatorname{det}(\Phi(z))=0$ satisfy $|z|>1$. Let

$$
\eta_{t}=B e_{t}
$$

be the $n \times 1$ vector of reduced form innovations, with (unconditional) covariance matrix $\Sigma_{\eta}=$ $B B^{\prime}$.

Suppose we are interested in the dynamic effects of the structural shocks in $e_{t}$. Let $A$ be the VAR companion matrix, $X_{t}^{c}:=\left(X_{t}^{\prime}, X_{t-1}^{\prime}, \ldots, X_{t-p+1}^{\prime}\right)^{\prime}$ the state vector associated with the VAR companion form and $R:=\left(I_{n}, 0_{n \times n}, \ldots, 0_{n \times n}\right)$ a selection matrix such that $X_{t}=R X_{t}^{c}, R R^{\prime}=I_{n}$. As is known, the dynamic response of $X_{t+h}$ to shock $e_{j t}$ to the variable $X_{j t}$ is summarized by the (population) IRF:

$$
I R F_{j}(h):=R(A)^{h} R^{\prime} b_{j} \quad, \quad h=0,1,2, \ldots, \quad j=1, \ldots, n
$$

where $b_{j}$ is the $j$-th column of $B$, i.e. $B:=\left(b_{\bullet j}: b_{j}: b_{j \bullet}\right)$, and $b_{\bullet j}$ and $b_{j \bullet}$ are the sub-matrices that contain the columns that precede (if any) and follow (if any) the column $b_{j}$, respectively. Absent further restrictions on the coefficients, the IRF in eq. (3) requires that $b_{j}$ is identified in the sense that it contains independent information relative to the columns in $b_{\bullet j}$ and/or in $b_{j \bullet}$. For $h=0$, the IRF in eq. (3) is such that, up to possible normalizations of the shocks, the 
element $b_{l j}$ of the $B$ matrix in eq. (2) captures the instantaneous (on-impact) effect of the $j$-th structural shock on the $l$-th variable of the system.

Consider now our specific case, where $n=3$. Let $Y_{t}$ denote a (scalar) measure of real activity, and let $U_{M t}$ and $U_{F t}$ be two (scalar) measures of macro and financial uncertainty, respectively, so that $X_{t}:=\left(U_{M t}, Y_{t}, U_{F t}\right)^{\prime}$. In the absence of further restrictions, the structural relationship in eq. (2) is given by the following system of equations

$$
\left(\begin{array}{c}
\eta_{M t} \\
\eta_{Y t} \\
\eta_{F t} \\
\eta_{t}
\end{array}\right)=\left(\begin{array}{ccc}
b_{M M} & b_{M Y} & b_{M F} \\
b_{Y M} & b_{Y Y} & b_{Y F} \\
b_{F M} & b_{F Y} & b_{F F}
\end{array}\right)\left(\begin{array}{c}
e_{M t} \\
e_{Y t} \\
e_{F t} \\
e_{t}
\end{array}\right)
$$

where we conventionally call $e_{M t}$ 'macroeconomic uncertainty shock', $e_{F t}$ 'financial uncertainty shock' and $e_{Y t}$ 'real economic activity shock'. As is known, at least three restrictions are needed in eq. (4) to identify the shocks in a 'Gaussian setup' 5 The covariance matrix $\Sigma_{\eta}=B B^{\prime}$ provides $n(n+1) / 2=6$ symmetry restrictions to identify the 9 elements of $B$, leaving 3 element unidentified. A common solution to this problem is to specify $B$ as a triangular matrix, which provides the 3 zero (identifying) restrictions. The empirical literature on the identification of uncertainty shocks largely relies on the use of recursive SVARs because the interest typically lies on the effect of uncertainty shocks on $Y_{t}$, while it is presumed that $U_{M t}\left(U_{F t}\right)$ responds to shocks to $Y_{t}$ only with lags. If one imposes an upper (lower) triangular structure on $B$, or 'conventional' zero restrictions, it is not possible to identify simultaneously the parameters of interest $b_{Y M}, b_{Y F}, b_{M Y}$ and $b_{F Y}$, meaning that 'reverse causality' cannot be addressed.

The reverse causality issue and the related identification problem can in principle be tackled by using valid external instruments that permit to increase the number of useful moment conditions other than $\Sigma_{\eta}=B B^{\prime}$, without further restricting $B$; see e.g. Stock and Watson (2012, 2018) and Mertens and Ravn (2013); see also Carriero et al. (2015). Ludvigson et al. (2018a) discuss the peril of such an approach in the uncertainty framework, and improve upon this methodology by arguing that if $U_{M t}$ and $U_{F t}$ are potentially endogenous (i.e. they may respond to $e_{Y t}$ ), then it is difficult to find credible observable exogenous external instruments for the uncertainty shocks.

While the combined use of external instruments and set-identification methods allow to address the reverse causality issue, it does not help dealing with the problem of possibly regime-

\footnotetext{
${ }^{5}$ It is worth stressing that regardless of the type of identifying restrictions we impose on $B$, we do not have enough information in this stylized small-scale model to claim that $e_{Y t}$ is a demand or supply shock. In general, $e_{Y t}$ could be a combination of technology, monetary policy, preferences and government expenditures. For this reason, and in line with Ludvigson et al. (2018a), we refer to $e_{Y t}$ as 'real activity shock'. Likewise, we do not have enough information to disentangle whether uncertainty shocks originate from economic policies and/or technology.
} 
dependent effects of uncertainty shocks, which is the issue analyzed next.

\subsection{Using heteroskedasticity to identify uncertainty and real economic activ- ity shocks}

In order to jointly address the reverse causality and possible regime-dependent effects of uncertainty shocks, one needs to combine a non-recursive structure for $B$ with the case where the elements in $B$ may change across macroeconomic regimes with the changes in the unconditional covariance matrix $\Sigma_{\eta}$, generating regime-dependent IRFs. We solve this problem by exploiting the heteroskedasticity displayed by the reduced form errors $\eta_{t}$ across different macroeconomic regimes that characterize the U.S. business cycle. Our identification methodology is based on the existence of different volatility regimes in the post-WW2 U.S. business cycle, i.e. different values that $\Sigma_{\eta}$ may take across sub-samples. Allowing for changes in the structural parameters $B$ represents a major generalization relative to the 'standard' identification approach based on heteroskedasticity developed in Rigobon (2003), Lanne and Lütkepohl (2008) and Lanne et al. (2010); see also Lewis (2018) ${ }^{6}$

Going back to the SVAR for $X_{t}:=\left(U_{M t}, Y_{t}, U_{F t}\right)^{\prime}$ defined in eq. (1), consider the unconditional covariance matrix $\Sigma_{\eta}$ :

$$
\Sigma_{\eta}=E\left(\eta_{t} \eta_{t}^{\prime}\right):=\left(\begin{array}{ccc}
\sigma_{M}^{2} & \sigma_{M, Y} & \sigma_{M, F} \\
& \sigma_{Y}^{2} & \sigma_{Y, F} \\
& & \sigma_{F}^{2}
\end{array}\right),
$$

where, $\sigma_{M, Y}=E\left(\eta_{M t} \eta_{Y t}\right), \sigma_{M, F}=E\left(\eta_{M t} \eta_{F t}\right)$ and $\sigma_{Y, F}=E\left(\eta_{Y t} \eta_{F t}\right)$. For ease of exposition, assume that there are two structural changes in this unconditional error covariance matrix, which correspond to the existence of three distinct volatility regimes $7^{7}$ If $t=T_{B_{1}}$ and $t=T_{B_{2}}$ denote the dates of the two structural breaks, with $1<T_{B_{1}}<T_{B_{2}}<T$, then the reduced form VAR in eq. (1) can be generalized to:

$$
X_{t}=\Pi(t) W_{t}+\eta_{t} \quad, \quad \Sigma_{\eta}(t):=E\left(\eta_{t} \eta_{t}^{\prime}\right), \quad t=1, \ldots, T
$$

\footnotetext{
${ }^{6}$ We refer to Lütkepohl (2013), Lütkepohl and Netšunajev (2017) and Kilian and Lütkepohl (2017, Chap. 14) for a review of this literature. Chen and Netšunajev (2018) provide an application of such methodology in the context of uncertainty shocks.

${ }^{7}$ This is the case we will deal with in our empirical section. Our analysis, however, can be easily generalized to the case in which there are $m$ structural breaks in the unconditional error covariance matrix, corresponding to $m+1$ volatility regimes in the data. The inferential issues that arise when the break dates are misspecified is a topic which has not been yet explicitly analyzed in the identification-through-heteroskedasticity literature, and is the subject of future research. Podstawki and Velinov (2018) have extended the identification approach we present and apply in this paper to the case in which the VAR parameters switch endogenously across volatility regimes.
} 
where $W_{t}:=\left(X_{t-1}^{\prime}, \ldots, X_{t-p}^{\prime}, 1\right)^{\prime}$ contains lagged regressors and a constant, $\Pi(t)$ is the matrix of associated slope (autoregressive) coefficients given by

$$
\Pi(t):=\Pi_{1} \times \mathbb{1}\left(t \leq T_{B_{1}}\right)+\Pi_{2} \times \mathbb{1}\left(T_{B_{1}}<t \leq T_{B_{2}}\right)+\Pi_{3} \times \mathbb{1}\left(t>T_{B_{2}}\right)
$$

and, finally, the error covariance matrix $\Sigma_{\eta}(t)$ is given by

$$
\Sigma_{\eta}(t):=\Sigma_{\eta, 1} \times \mathbb{1}\left(t \leq T_{B_{1}}\right)+\Sigma_{\eta, 2} \times \mathbb{1}\left(T_{B_{1}}<t \leq T_{B_{2}}\right)+\Sigma_{\eta, 3} \times \mathbb{1}\left(t>T_{B_{2}}\right)
$$

where $\mathbb{1}(\cdot)$ is the indicator function. Key to our identification approach is that $\Sigma_{\eta, 1} \neq \Sigma_{\eta, 2} \neq$ $\Sigma_{\eta, 3}$. Important for our analysis, notice that the specification in eq.s (6)-(8) covers the case in which also the slope (autoregressive) parameters vary across volatility regimes $\left(\Pi_{1} \neq \Pi_{2} \neq \Pi_{3}\right)$.

We assume that the system described by eq.s (6)-(8) is subject to a set of regularity assumptions (Assumptions 1-3 in the Technical Supplement) which allow standard inference. Given the existence of three volatility regimes, the SVAR is defined by the structural specification:

$$
\begin{array}{lr}
\eta_{t}=B e_{t} & 1 \leq t \leq T_{B_{1}} \\
\eta_{t}=\left(B+Q_{2}\right) e_{t} & T_{B_{1}}<t \leq T_{B_{2}} \\
\eta_{t}=\left(B+Q_{2}+Q_{3}\right) e_{t} & T_{B_{2}}<t \leq T
\end{array}
$$

where $B, Q_{2}$ and $Q_{3}$ are $3 \times 3$ matrices containing structural parameters and $e_{t}:=\left(e_{M t}, e_{Y t}, e_{F t}\right)^{\prime}$ is the vector of structural shocks such that $E\left(e_{t}\right)=0_{3 \times 1}$ and with normalized covariance matrix $E\left(e_{t} e_{t}^{\prime}\right):=I_{3} \mathrm{~B}^{8}$ As before, we call $e_{M t}$ 'macroeconomic uncertainty shock', $e_{F t}$ 'financial uncertainty shock' and $e_{Y t}$ 'shock to real activity'. In eq. (9), $B$ is the non-singular matrix which governs the structural contemporaneous relationships (on-impact responses) between the variables and the shocks in the first volatility regime. The matrix $Q_{2}$ captures the changes in the structural parameters, if any, from the first to the second volatility regime, hence the non-singular matrix $\left(B+Q_{2}\right)$ captures the structural contemporaneous relationship (on-impact responses) between the variables and the shocks in the second volatility regime. The matrix $Q_{3}$ captures the change in the structural parameters, if any, from the second to the third volatility regime, hence the non-singular matrix $\left(B+Q_{2}+Q_{3}\right)$ captures the structural relationship (on-impact responses) between the variables and the shocks in the third volatility regime.

\footnotetext{
${ }^{8}$ An alternative and equivalent parametrization of the SVAR in eq. 9 is discussed in the Technical Supplement, and is based on the assumptions that the structural shocks have a diagonal matrix covariance matrix which changes across volatility regimes, i.e. $E\left(e_{i, t} e_{i, t}^{\prime}\right):=\Lambda_{i}:=\operatorname{diag}\left(\lambda_{i, 1}, \ldots, \lambda_{i, n}\right)$, where $e_{i, t}$ is the vector of structural shocks at time $t$ in the regime volatility $i$, and $\lambda_{i, j}$ is the variance of the structural shock to variable $j$ in the volatility regime $i$. The IRFs presented and discussed in eq. 15 below can be 'scaled' accordingly. To keep exposition as simple as possible, in the paper we refer, without loss of generality, to the parametrization of the SVAR in eq. 9 .
} 
Eq. (9) leads to the system of second-order moment conditions

$$
\begin{aligned}
\Sigma_{\eta, 1} & =B B^{\prime} \\
\Sigma_{\eta, 2} & =\left(B+Q_{2}\right)\left(B+Q_{2}\right)^{\prime} \\
\Sigma_{\eta, 3} & =\left(B+Q_{2}+Q_{3}\right)\left(B+Q_{2}+Q_{3}\right)^{\prime}
\end{aligned}
$$

which link the reduced form to the structural parameters. Equations 10$)-(12)$ provide $r=$ $\frac{3}{2} n(n+1)$ identifying restrictions on $B, Q_{2}$ and $Q_{3}$ induced by symmetry. The total number of elements in $B, Q_{2}$ and $Q_{3}$ is $3 n^{2}$, hence it is necessary to impose at least $3 n^{2}-r$ additional constraints to achieve identification. These $3 n^{2}-r$ identifying constraints are provided by economic reasoning about the way the on-impact coefficients may change across regimes, which means that the suggested identification approach combines both data properties (i.e. the heteroskedasticity provided by the data) and theoretical considerations reflected in the specification of the structure of the matrices $B,\left(B+Q_{2}\right)$ and $\left(B+Q_{2}+Q_{3}\right)$. Let $\psi$ be the vector defined as $\psi:=\left(\operatorname{vec}(B)^{\prime}, \operatorname{vec}\left(Q_{2}\right)^{\prime}, \operatorname{vec}\left(Q_{3}\right)^{\prime}\right)^{\prime}$. The set of theory-based linear identifying restrictions on $B$, $Q_{2}$ and $Q_{3}$ can be represented compactly in explicit form by:

$$
\psi=G \theta+d
$$

where $\theta$ is the vector containing the 'free' elements in $B, Q_{2}$ and $Q_{3}, G$ is a known $3 n^{2} \times \operatorname{dim}(\theta)$ selection matrix of full column rank, $d:=\left(d_{B}^{\prime}, d_{Q_{2}}^{\prime}, d_{Q_{3}}^{\prime}\right)^{\prime}$ is a $3 n^{2} \times 1$ vector containing known elements.$^{9}$ The moment conditions in eq.s $107-12$ along with the constraints in eq. 13 can be conveniently summarized in the expression

$$
\sigma^{+}=g(\theta)
$$

where $\sigma^{+}:=\left(\operatorname{vech}\left(\Sigma_{\eta, 1}\right)^{\prime}, \operatorname{vech}\left(\Sigma_{\eta, 2}\right)^{\prime}, \operatorname{vech}\left(\Sigma_{\eta, 3}\right)^{\prime}\right)^{\prime}$ is $r \times 1$, and $g(\cdot)$ is a nonlinear (differentiable) vector function (see Bacchiocchi and Fanelli, 2015 for details). It turns out that the necessary and sufficient rank condition for identification is that the Jacobian matrix $J(\theta):=\frac{\partial g(\theta)}{\partial \theta^{\prime}}$ be regular and of full column rank when evaluated in a neighborhood of the true parameter value $\theta_{0}$. The necessary order condition is $\operatorname{dim}(\theta) \leq r$. The Jacobian $J(\theta)$ can be derived analytically or evaluated numerically. Thus, in order to identify the shocks it is necessary that the restrictions in eq.s 10)-13) satisfy also the necessary and sufficient rank condition.

We denote with $\tilde{B}=B(\theta), \tilde{Q}_{2}=Q_{2}(\theta)$ and $\tilde{Q}_{3}=Q_{3}(\theta)$ the counterparts of $B, Q_{2}$ and $Q_{3}$ which fulfill the identification conditions. Interestingly, $\tilde{B}$ (first regime), $\left(\tilde{B}+\tilde{Q}_{2}\right)$ (second

\footnotetext{
${ }^{9}$ Other than accounting for (possibly) non-homogeneous restrictions (meaning that the vector $d$ can be nonzero), eq. (13) allows for cross-regime constraints, i.e. simultaneous restrictions which involve the elements of the matrices $B, Q_{2}$ and $Q_{3}$ like, for example, $b_{12}+q_{2,12}=0$ or $b_{12}+q_{2,12}+q_{2,12}=1$, where $b_{12}, q_{2,12}$ and $q_{3,12}$ are the $(1,2)$ elements of $B, Q_{2}$ and $Q_{3}$, respectively.
} 
regime) and $\left(\tilde{B}+\tilde{Q}_{2}+\tilde{Q}_{3}\right)$ (third regime), may be either triangular or 'full' depending on the specification at hand, therefore reverse causality phenomena can in principle be modeled. Notably, in this setup overidentified SVARs, i.e. those for which $\operatorname{dim}(\theta)<r$, can be tested against the data.

The so-identified SVAR generates regime-dependent IRFs. Let $A_{i}, i=1,2,3$ be the reduced form companion matrices associated with the system in eq. (6). The dynamic response of $X_{t+h}$ to a one-standard deviation shock in variable $j$ at time $t$ is summarized by the (population) IRFs:

$$
I R F_{j}(h):=\left\{\begin{array}{llc}
R^{\prime}\left(A_{1}\right)^{h} R \tilde{b}_{j} & t \leq T_{B_{1}} \\
R^{\prime}\left(A_{2}\right)^{h} R\left(\tilde{b}_{j}+\tilde{q}_{2 j}\right) & T_{B_{1}}<t \leq T_{B_{2}} & h=0,1, \ldots, h_{\max } \\
R^{\prime}\left(A_{3}\right)^{h} R\left(\tilde{b}_{j}+\tilde{q}_{2 j}+\tilde{q}_{3 j}\right) & t>T_{B_{2}} & j=M, Y, F
\end{array}\right.
$$

where $R$ is the selection matrix introduced in Section 2.1, $\tilde{b}_{j}$ is the $j$-th column of the matrix $\tilde{B}$, $\tilde{b}_{j}+\tilde{q}_{2 j}$ is the $j$-th column of the matrix $\tilde{B}+\tilde{Q}_{2}, \tilde{b}_{j}+\tilde{q}_{2 j}+\tilde{q}_{3 j}$ is the $j$-th column of the matrix $\tilde{B}+\tilde{Q}_{2}+\tilde{Q}_{3}$, respectively, and $h_{\max }$ is the largest horizon considered. Even in the special case in which the slope (autoregressive) coefficients do not vary across volatility regimes, i.e. when $A_{1}=A_{2}=A_{3}$ (meaning that $\Pi_{1}=\Pi_{2}=\Pi_{3}$ in eq. (7)), the IRFs in eq. (15) change across volatility regimes because of the changes in the on-impact response coefficients.

\section{Model specification and empirical results}

In this section, we apply the SVAR for $X_{t}:=\left(U_{M t}, Y_{t}, U_{F t}\right)^{\prime}$ presented in eq. (1) and discussed in the previous section to address our two main research questions: (i) Does the response of $Y_{t}$ to shocks to $\left(U_{M t}, U_{F t}\right)$ vary across macroeconomic regimes? (ii) Are $U_{M t}$ and $U_{F t}$ exogenous sources of fluctuations in $Y_{t}$, or do $U_{M t}$ and $U_{F t}$ respond endogenously to shocks in $Y_{t}$ ? In Section 3.1 we present the data and in Section 3.2 we provide evidence for the existence of three broad volatility regimes. In Section 3.3 we specify and discuss the baseline non-recursive SVAR and in Section 3.4 we test for exogenous uncertainty and analyze the resultant IRFs.

\subsection{Data}

Our VAR includes three variables; $U_{M t}(f), U_{F t}(f)$ and $Y_{t}$, where $Y_{t}$ is a measure of real economic activity, $U_{M t}(f)$ is a measure of $f$-period-ahead macroeconomic uncertainty and $U_{F t}(f)$ is a measure of $f$-period-ahead financial uncertainty, where $f=1$ (one-month) or $f=12$ (one-year). Our measure of real economic activity is the growth rate of the log of real industrial production, denoted $\Delta i p_{t}$. The real industrial production index is taken from the FRED database. The measure of financial uncertainty is taken from Ludvigson et al. (2018a), while the index of 
macroeconomic uncertainty is taken from Jurado et al. (2015) 10 The data are monthly and cover the period 1960M8-2015M4 for a total of $T=653$ observations. As discussed in Ludvigson et al. (2018a), jointly modeling financial and macroeconomic uncertainty is key to obtain a correct understanding of the relationship between uncertainty and the business cycle.

\subsection{Volatility breaks}

Two crucial features of our VAR are that the identification approach requires breaks in the unconditional volatility of the data, and that a small-scale system like ours is not affected by nonfundamentalness, which implicitly amounts to claim that it does not omit important variables. Our major hypothesis is that the relationship between uncertainty and real activity vary across the main macroeconomic regimes of post-WW2 U.S. business cycle because of changes in the unconditional variance of $Y_{t}$. To provide evidence in favour of volatility breaks, we proceed in two steps. First, we provide suggestive evidence of time variation by looking at recursive and rolling windows estimates of the residual variances and covariances in our baseline VAR. Second, we formally test for the existence of two structural breaks using Chow-type tests, with possible break dates identified in the previous step. Next, we deal with potential nonfundamentalness of our VAR by testing for its 'informational sufficiency' using the procedure by Forni and Gambetti (2014) and factors extracted from the McCracken and Ng (2015)'s large set of macroeconomic and financial variables. The detailed investigation of this last issue, sketched in the Technical Supplement, is important in light of the small dimension of $X_{t}:=\left(U_{M t}, Y_{t}, U_{F t}\right)^{\prime}$ because nonfundamentalness is best seen as an informational deficiency problem. The empirical analysis shows that we do not reject the informational sufficiency of $X_{t}:=\left(U_{M t}, Y_{t}, U_{F t}\right)^{\prime}$, meaning that we can correctly estimate the effects of uncertainty shocks through IRFs.

We start by estimating our baseline VAR for $X_{t}:=\left(U_{M t}, Y_{t}, U_{F t}\right)^{\prime}$ with four lags $(p=4)$ both recursively and over 10- and 15-years rolling-windows. The estimates of the six elements of the unconditional VAR error covariance matrix $\Sigma_{\eta}$ are plotted in Figure 1. The graphs on the diagonal report the estimated variances while the off-diagonal terms report the estimated covariances for the recursive (blue line), the 10-years (red line) and the 15-years (yellow line) rolling windows VARs. The graph in the position $(2,2)$ reports the unconditional variance of the residuals of the second equation of our VAR, the one associated with $Y_{t}$, i.e. $\sigma_{Y}^{2}$ in $\Sigma_{\eta}$ in eq. (5). The graph clearly shows that the average volatility level is time-varying, being higher during the seventies and eighties, declining from the mid-eighties until the end of 2007, and then increasing again after the financial crisis of 2007-08 before stabilizing. All the remaining graphs in Figure 1 broadly confirm the presence of three volatility regimes. As expected, the two main changes of

\footnotetext{
${ }^{10}$ The Technical Supplement discusses at length how the two proxies of uncertainty have been constructed.
} 
volatility occur in correspondence of the beginning of the Great Moderation and Great Recession periods, respectively. The two dashed vertical lines correspond to the possible break dates, i.e. $T_{B_{1}}=1984 \mathrm{M} 3$ and $T_{B_{2}}=2007 \mathrm{M} 12$. These two break dates would partition the whole sample period 1960M8-2015M4 into three different sub-samples: the Great Inflation period (1960M81984M3, $T=280$ ), the Great Moderation period (1984M4-2007M12, $T=285)$, and the Great Recession+Slow Recovery period (2008M1-2015M4, $T=88)$ 11 It is worth noting, however, that while the unconditional variance associated with the proxy of macroeconomic uncertainty roughly follows the same volatility pattern as the unconditional volatility of $Y_{t}$ (position $(1,1)$ in Figure 1), the unconditional variance associated with the proxy of financial uncertainty increases until the beginning of the nineties, probably because of the process of financial innovation which characterizes U.S. financial markets (position (3,3) in Figure 1). Interestingly, these differences in volatility patterns provide identification information in our approach.

The evidence reported in Figure 1 is broadly consistent with the information conveyed in Table 112 The second column of Table 1 summarizes the OLS-based estimates of the VAR covariance matrix $\Sigma_{\eta}$ on the whole sample, i.e. under the null hypothesis that there are no volatility regimes in the data $\left(H_{0}^{\prime}: \Sigma_{\eta, 1}=\Sigma_{\eta, 2}=\Sigma_{\eta, 3}\right)$, and then separately on the three volatility sub-periods 13 As already shown in Figure 1, these results confirm that unconditional variances and covariances have changed over time. Table 1 also summarizes some diagnostic statistics associated with the estimated models, which suggest that VAR residuals tend to be not Gaussian but not serially correlated within regimes. The non-normality of VAR disturbances is detected, as expected, on the overall sample period but also within macroeconomic regimes and is fully consistent with the analysis in e.g. Cúrdia et al. (2014). We remark that the possible

\footnotetext{
${ }^{11}$ As concerns the third volatility regime, according to the U.S. National Bureau of Economic Research the Great Recession began in December 2007 and ended in June 2009, thus extending over 19 months. Thus, we treat $T_{B_{2}}=2007 \mathrm{M} 12$ as the date in which the Great Moderation ends. Considering three distinct volatility regimes does not necessarily rule out the possibility that the VAR for $X_{t}:=\left(U_{M t}, Y_{t}, U_{F t}\right)^{\prime}$ might display unconditional (or possibly conditional) heteroskedastic disturbances within regimes, other than across them. This is clearly seen from the graphs in Figure 1 but, as discussed below, does not represent a major obstacle to the implementation of our identification approach.

${ }^{12}$ Admittedly, the evidence in Figure 1 could also support a time-varying specification. We refer to Mumtaz and Theodoridis (2018) and Carriero et al. (2018a, 2018b) for different views on how time-varying specifications can be fruitfully exploited to address empirically the role of uncertainty. As already stressed, crucial to our identification approach is the existence of broad volatility regimes in the data.

${ }^{13}$ The OLS estimates in Table 1 correspond to maximum likelihood estimates generated by maximizing Gaussian densities within each of the considered samples. In the Technical Supplement, we also discuss a classical minimum distance (CMD) estimation approach which does not require any distributional assumption. We prefer to stick to Gaussian maximum likelihod estimation of our SVAR to be as close as possible to the more familar identificationthrough-heteroskedasticiy approach put forth by Lanne and Lütkepohl (2008) in the context of SVARs.
} 
presence of within-regimes heteroskedasticity (conditional or unconditional), while affecting the full efficiency of our estimates, does not represent a major obstacle to the identification strategy presented below.

To verify formally the hypothesis that there are two main structural breaks in the VAR error covariance matrix at the dates $T_{B_{1}}=1984 \mathrm{M} 3$ and $T_{B_{2}}=2007 \mathrm{M} 12$, we compute a set of Chow-type tests and misspecification-type tests. We first test whether the joint null hypothesis of absence of structural breaks in all VAR coefficients:

$$
H_{0}:\left(\begin{array}{c}
\Pi_{1} \\
\Sigma_{\eta, 1}
\end{array}\right)=\left(\begin{array}{c}
\Pi_{2} \\
\Sigma_{\eta, 2}
\end{array}\right)=\left(\begin{array}{c}
\Pi_{3} \\
\Sigma_{\eta, 3}
\end{array}\right)=\left(\begin{array}{c}
\Pi \\
\Sigma_{\eta}
\end{array}\right)
$$

is rejected and, conditional on the rejection of $H_{0}$, we test the null hypothesis of absence of volatility regimes

$$
H_{0}^{\prime}: \Sigma_{\eta, 1}=\Sigma_{\eta, 2}=\Sigma_{\eta, 3}
$$

under the maintained restriction: $\Pi_{1}=\Pi_{2}=\Pi_{3}=\Pi$ on slope coefficients. Results are summarized in the bottom panel of Table 1 which reports the LR tests for the hypotheses $H_{0}$ and $H_{0}^{\prime}$, respectively. Both $H_{0}$ and $H_{0}^{\prime}$ are strongly rejected by the data. As a final check, we investigate to what extent the detected regime-dependence in the residual covariance matrix can be ascribed to the regime-dependence that characterizes the autoregressive parameters. To do so, we estimate the VAR in eq. (6) by allowing the autoregressive parameters to change as in eq. (7) with $T_{B_{1}}=1984 \mathrm{M} 3$ and $T_{B_{2}}=2007 \mathrm{M} 12$, keeping the covariance matrix $\Sigma_{\eta}$ constant. In the so-estimated model, we perform a test for the null hypothesis of (unconditional) homoskedasticity in the residuals $\left(H_{0}^{\prime \prime}\right)$, which is reported in the lower panel of Table 1 . Results show that the hypothesis of homoskedasticity is strongly rejected by the data. This evidence confirms that the changes that characterize the unconditional covariance matrix $\Sigma_{\eta}$ can not be solely ascribed to the changes in the autoregressive parameters. Overall, our results are consistent with Aastveit et al. (2017) who, using a wide range of econometric techniques, provide substantial evidence against the stability of common VARs in the period since the Great Recession.

Other than documenting the existence of three broad volatility regimes in the data, Table 1 provides some rough evidence about the changing nature of the relationships between our proxies of uncertainty, $U_{M t}$ and $U_{F t}$, and real economic activity, $Y_{t}$. Although it is not possible to infer any causality direction from the correlations in Table 1, the data clearly point towards changing relationships. The information provided by the correlations in Table 1 will be used to inform the structural specification in the next section.

Overall, the estimated reduced form system for $X_{t}:=\left(U_{M t}, Y_{t}, U_{F t}\right)^{\prime}$ provides a reasonable fit to the data and is 'informational sufficient'. We consider it a statistically satisfactory reduced form representation of the non-recursive SVAR specified next. 


\subsection{Non-recursive SVAR specification}

In this section we discuss the specification of the matrices of structural parameters $\tilde{B}=B(\theta)$, $\tilde{Q}_{2}=Q_{2}(\theta)$ and $\tilde{Q}_{3}=Q_{3}(\theta)$ in eq. (9). The vector of structural shocks is $e_{t}:=\left(e_{M t}, e_{Y t}, e_{F t}\right)^{\prime}$, and we call conventionally $e_{M t}$ 'macroeconomic uncertainty shock', $e_{F t}$ 'financial uncertainty shock' and $e_{Y t}$ 'real activity shock', see the discussion in Section 2.

To inform the structural specification, valuable indications may be inferred from the VAR residuals correlation matrices sketched in Table 1. Three main empirical facts emerge from this table. First, the negative correlation between (the residuals associated with) macroeconomic uncertainty and industrial production growth increases by about $50 \%$, from $-14 \%$ to $-21 \%$, when moving from the Great Moderation to the Great Recession+Slow Recovery period. This reduced form evidence is consistent with the structural analysis in e.g. Caggiano et al. (2017a) and Plante et al. (2018). Second, the correlation between (the residuals associated with) financial uncertainty and industrial production growth turns negative only in the Great Recession+Slow Recovery period $(3.8 \%, 3.2 \%$ and $-8.9 \%$, respectively) and is not significant. Third, the correlation between (the residuals associated with) macroeconomic and financial uncertainty increases substantially across the three volatility regimes $(12 \%, 32 \%$ and almost $40 \%$, respectively), suggesting that the two sources of uncertainty developed in a relatively independent way during the Great Inflation period, and started to be much more correlated thereafter, when periods of financial turmoil have become more prominent. These three empirical facts suggest that the (negative) relationship between macroeconomic uncertainty and real economic activity is likely regime-dependent and intensifies after the GFC. On the other hand, the channel which connects financial uncertainty and real economic activity appears to be indirect: since financial uncertainty is virtually uncorrelated with real activity, its effects on the business cycle, if any, might work only via its correlation with macroeconomic uncertainty. This latter correlation is almost irrelevant in the first subsample, and increases only after the mid-1980s.

Based on these considerations, we formulate our hypotheses on the structural parameters. The three volatility regimes detected in the previous section provide us with $r=3 / 2(n)(n+$ 1) $=18$ moment conditions. In the absence of restrictions, $B, Q_{2}$ and $Q_{3}$ contain $3 n^{2}=27$ elements, hence it is necessary to place at least $3 n^{2}-r=9$ parameter constraints on these matrices in order to achieve identification. These restrictions, which can be represented compactly as in eq. (13), must satisfy the necessary and sufficient identification rank condition discussed in Section 2.2, i.e. the Jacobian matrix associated with the function in eq. 14 must be regular and full column rank. We consider a total of 11 identifying restrictions (which lead to 11-9=2 
overidentification restrictions) and the following matrices $\tilde{B}, \tilde{B}+\tilde{Q}_{2}$ and $\tilde{B}+\tilde{Q}_{2}+\tilde{Q}_{3}$ :

Great Inflation:

$$
\tilde{B}:=\left(\begin{array}{ccc}
b_{M M} & b_{M Y} & 0 \\
b_{Y M} & b_{Y Y} & 0 \\
0 & 0 & b_{F F}
\end{array}\right)
$$

Great Moderation:

$$
\tilde{B}+\tilde{Q}_{2}:=\left(\begin{array}{ccc}
b_{M M}+q_{2, M M} & b_{M Y} & q_{2, M F} \\
b_{Y M}+q_{2, Y M} & b_{Y Y}+q_{2, Y Y} & 0 \\
q_{2, F M} & 0 & b_{F F}+q_{2, F F}
\end{array}\right)
$$

Great Recession + Slow Recovery:

$$
\tilde{B}+\tilde{Q}_{2}+\tilde{Q}_{3}:=\left(\begin{array}{ccc}
b_{M M}+q_{2, M M} & b_{M Y} & q_{2, M F}+q_{3, M F} \\
b_{Y M}+q_{2, Y M}+q_{3, Y M} & b_{Y Y}+q_{2, Y Y}+q_{3, Y Y} & q_{3, Y F} \\
q_{2, F M} & 0 & b_{F F}+q_{2, F F}+q_{3, F F}
\end{array}\right) \text {, }
$$

so that the vector of structural parameters $\theta$ contains 16 non-zero elements $(\operatorname{dim}(\theta)=16)$.

The specification of the matrix $\tilde{B}$ (Great Inflation) in eq. 18 is based on one crucial hypothesis. Inspired by Ng and Wright (2013) and the already commented reduced-form evidence in Table 1, we maintain that heavily regulated financial markets before the 1980s slowed down the response of financial markets to non-financial dynamics on the one hand, and the response of macroeconomic variables to the uncertainty generated by financial markets on the other hand. Thus, financial uncertainty is assumed not to respond on-impact to real activity shocks $\left(b_{F Y}=0\right)$ nor to macro uncertainty shocks $\left(b_{F M}=0\right)$, and real activity is assumed not to respond on-impact to financial uncertainty shocks $\left(b_{Y F}=0\right)$, though lagged responses are not ruled out and depend on the estimated dynamics. Likewise, it is also assumed that financial uncertainty does not exert contemporaneous effects on macroeconomic uncertainty $\left(b_{M F}=0\right)$. Overall, according to the $\tilde{B}$ matrix in eq. 18, macroeconomic uncertainty can be potentially endogenous, depending on the significance of the parameter $b_{M Y}$, while financial uncertainty is treated as a variable that can react only with lags.

Moving to the second volatility regime (Great Moderation), the non-recursive structure of the matrix $\tilde{B}+\tilde{Q}_{2}$ in eq. 18 is still consistent with the idea that macroeconomic uncertainty shocks may affect real economic activity instantaneously through the parameter $b_{Y M}+q_{2, Y M}\left(q_{2, Y M}\right.$ captures the change of impact relative to the Great Inflation) and, in turn, real activity shocks may affect macro uncertainty through the parameter $b_{M Y}+q_{2, M Y}$ (again, $q_{2, M Y}$ captures the change relative to the Great Inflation). Differently from the Great Inflation period, however, we now admit that causation among the two sources of uncertainty may run both ways through the 
parameters $q_{2, F M}$ (position $\left.(3,1)\right)$ and $q_{2, M F}$ (position $(1,3)$ ), respectively. This is done to infer whether the increased correlation between $U_{M t}$ and $U_{F t}$ observed during the Great Moderation relative to the Great Inflation can be ascribed to financial or macro uncertainty shocks, or to both types of shocks. For instance, with $q_{2, F M}=0$ and $q_{2, M F} \neq 0\left(q_{2, M F}>0\right)$ in eq. (18) we might conclude that causality runs from financial uncertainty shocks to macroeconomic uncertainty alone.

Finally, the causality relationships entailed by the structure of the matrix $\tilde{B}+\tilde{Q}_{2}+\tilde{Q}_{3}$ (Great Recession+Slow Recovery) in eq. (18) is similar to that of the Great Moderation, the main difference being that we now allow financial uncertainty shocks to affect real economic activity both directly (through the parameter $q_{3, Y F}$ ) and indirectly through its effect on macroeconomic uncertainty (through the parameter $q_{2, M F}+q_{3, M F}$ where, recall, $q_{3, M F}$ captures the possible change of effect relative to the Great Moderation).

Overall, the SVAR based on the specification in eq. (18) is identified in the sense that it satisfies the rank condition discussed in Section 2.2, and gives rise to $r-\operatorname{dim}(\theta)=2$ (testable) overidentification restrictions. Financial uncertainty is given the 'passive' role of merely amplifying the shocks before the 1980s, while the role of financial markets and the uncertainty stemming from them are brought back to the center-stage of business cycle after the mid-1980s 14 Notably, the specified structural model features possibly endogenous uncertainty, since it allows the structural parameter $b_{M Y}$ to be non-zero. Hence, testing $b_{M Y}=0$ amounts to testing for exogeneity of macroeconomic uncertainty.

Our testing procedure compares the specification in eq. (18) with a restricted version which features two additional hypotheses about the pass-through from uncertainty to real economic activity: one is the hypothesis of 'exogenous' macroeconomic uncertainty, $b_{M Y}=0$, and the other is the hypothesis that macroeconomic uncertainty shocks do not trigger financial uncertainty, $q_{2, F M}=0$, so that their structural relationship is unidirectional. Jointly, the two restrictions

$$
\begin{array}{cc}
b_{M Y}=0 & \text { 'exogenous' macro uncertainty } \\
q_{2, F M}=0 & \text { 'one-way' causality from financial to macro uncertainty }
\end{array}
$$

imply, when imposed in eq. (18), an overidentified system which features $r-\operatorname{dim}(\theta)=4$ (testable) overidentification restrictions.

\footnotetext{
${ }^{14}$ Our choice is also supported by institutional facts, in particular the changes in the norms regulating financial markets which occurred in the early 1980s, like the Depository Institutions Deregulation and Monetary Control Act in 1980, particularly the termination of regulation Q, and the Garn-St. Germain Act of 1982, which granted easier access to financial liquidity to households and firms only from the mid-eighties onwards.
} 


\subsection{On-impact and dynamic causal effects}

The non-recursive SVAR specified in eq. (18) is based on the idea that the changes in the covariance matrices $\Sigma_{\eta, 1} \neq \Sigma_{\eta, 2} \neq \Sigma_{\eta, 3}$ associated with the break dates $T_{B_{1}}=1984 \mathrm{M} 3$ and $T_{B_{2}}=2007 \mathrm{M} 12$, are explained by the occurrence of breaks in the structural parameters. As already observed in Section 2.2, the main difference between our approach and Lanne and Lütkepohl (2008)'s approach is that in the latter the autoregressive parameters in eq. (7) are kept constant, and the changes in the unconditional covariance matrix in the three volatility regimes are modelled by the simultaneous diagonalization:

$$
\Sigma_{\eta, 1}=B B^{\prime} \quad, \quad \Sigma_{\eta, 2}=B \Lambda_{2} B^{\prime} \quad, \quad \Sigma_{\eta, 3}=B \Lambda_{3} B
$$

where the elements of $B$ are fixed, $\Lambda_{2} \neq \Lambda_{3} \neq I_{n}$ are two diagonal matrices with positive elements on the diagonal which satisfy a set of identification conditions discussed in detail in Lanne et al. (2010). Eq. 20) gives rise to dynamic causal effects which are invariant to volatility regimes. Hence, before moving to the estimation of our structural model, it seems natural to test to what extent the specification in eq. (20) is supported/rejected by the data. The model in eq. (20) entails an overidentified system which incorporates three (testable) restrictions (indeed there are $r=18$ reduced form covariance parameters and $9+6=15$ distinct elements in $B, \Lambda_{2}$ and $\Lambda_{3}$ ). The likelihood ratio test for the overidentification restrictions implied by the specification in eq. 20 is equal to 10.11 with associated p-value of 0.0176 , hence the model is rejected at the $5 \%$ level of significance. We interpret this result as supportive of the fact that the onimpact coefficients of SVARs in the uncertainty framework can possibly change across major macroeconomic regimes of the U.S. economic history. In the analysis that follows, in order to highlight the importance of regime-dependent coefficients, the IRFs implied by the SVAR in eq. (20) will serve as comparative benchmark against our model.

The non-recursive SVAR specified in eq. (18) is estimated on the period 1960M8-2015M4 by imposing the three volatility regimes associated with the two break dates $T_{B_{1}}=1984 \mathrm{M} 3$ and $T_{B_{2}}=2007 \mathrm{M} 12$. The (quasi-)maximum likelihood estimates of the structural parameters $\theta$ that enter the matrices $\tilde{B}, \tilde{B}+\tilde{Q}_{2}$ and $\tilde{B}+\tilde{Q}_{2}+\tilde{Q}_{3}$ are reported, for $f=1$ (one-month uncertainty), in Table 2, along with analytic and bootstrap standard errors ${ }^{15}$ The upper panel

\footnotetext{
${ }^{15}$ Bootstrap standard errors are computed using Kilian's (1998) bootstrap-after-bootstrap method, keeping the break dates $T_{B_{1}}=1984 \mathrm{M} 3$ and $T_{B_{2}}=2007 \mathrm{M} 12$ fixed and resampling (non-parametrically) separately within each volatility regime. This method is also used to compute $90 \%$ bootstrap confidence bands for the IRFs that follow. Brüggemann et al. (2016) have shown that estimation uncertainty in IRFs produced by SVARs may increase dramatically in the presence of conditional heteroskedasticity compared to an i.i.d setup, depending especially on the persistence characterizing the underlying conditional heteroskedasticity processes. In our setup, the occurrence of conditional heteroskedasticity within the three volatility regimes is an issue which can not be
} 
of Table 2 refers to the the specification in eq. (18), while the lower panel refers to the same model estimated under the two additional restrictions in eq. (19). The estimated structural parameters $\hat{\theta}$ in Table 2 correspond to the on-impact responses featured by our IRFs.

We first discuss the reverse causality/exogeneity issue, then we analyze the dynamic causal effects implied by the estimated IRFs.

Reverse causality/exogeneity. The estimates in the two panels of Table 2 deliver an answer to our first research question, i.e. whether macroeconomic uncertainty is an exogenous source of economic fluctuations or an endogenous response to it, or both. We first analyze the model in the upper panel of Table 2, which allows for 'endogenous' macroeconomic uncertainty $\left(b_{M Y} \neq 0\right)$ and bidirectional causality between macroeconomic and financial uncertainty from the Great Moderation onwards $\left(q_{2, F M} \neq 0, q_{2, M F} \neq 0, q_{3, M F} \neq 0\right)$. The LR test for the two overidentification restrictions featured by this model is equal to 7.35 and has a p-value of 0.025 , hence the model is not supported by the data at the $5 \%$ level of significance.

The parameter $b_{M Y}$, which captures the on-impact response of macroeconomic uncertainty to real economic activity shocks in the three volatility regimes, is not statistically significant. The hypothesis of 'exogenous' macroeconomic uncertainty is largely supported by the data as the LR test for $b_{M Y}=0$ is equal to 0.056 and has a p-value of 0.94 . The estimated parameter $q_{2, F M}$ proves to be not strongly significant, confirming our intuition that since the 1980s the passthrough between the two sources of uncertainty is unidirectional: from financial uncertainty shocks to macroeconomic uncertainty. The estimated structural model in the lower panel of Table 2 incorporates these two additional restrictions, see eq. (19). In this case, the LR test for the four overidentification restrictions featured by the SVAR is equal to 8.03 with associated p-value of 0.091, which does not lead us to reject the model at the $5 \%$ significance level. A LR test for the structural model in the lower panel against the one in the upper panel of Table 2 is equal to 0.672 and has p-value equal to 0.713 . Overall, our empirical evidence supports the specification in eq.s $18-19 \cdot 16$

ruled out a priori, given the diagnostic tests in Table 1. Brüggemann et al. (2016) have also shown that the residual-based moving block bootstrap results in asymptotically valid inference, see also Kilian and Lütkepohl (2017, Ch. 12). Since available simulation results suggest that the performance of different bootstrap methods is often hardly distinguishable in finite samples, Brüggemann et al. (2016) recommend that practitioners should be aware of the fact that reported impulse response intervals may understate the actual estimation uncertainty in the presence of conditional heteroskedasticity. With this in mind, and in the absence of a detailed quantification of the extent of conditional heteroskedasticity in our SVAR (which is beyond the scopes of this paper), we interpret all reported bootstrap confidence bands for IRFs with caution.

${ }^{16}$ As a Referee has pointed out, the increase of the p-value that characterizes the two LR tests can also be explained by the fact that the latter can be less powerful than the former due to the increased number 
It could be argued that macroeconomic and financial uncertainty are treated asymmetrically in our model. Indeed, although the reduced form evidence speaks loudly about the role of financial uncertainty, the non-response of financial uncertainty to real economic activity shocks has been imposed in the structural specification. To address this issue, we re-estimate the SVARs in eq.s 18$)-19$ by inverting the positions of $U_{M, t}$ and $U_{F, t}$ in the vector $X_{t}$. This leads to a radical change in the role played by the two sources of uncertainty in the system and the way they transmit to the business cycle. In this case, the LR test for the overidentification restrictions is equal to 28.47 with a p-value of 0.00 , which strongly rejects the model. We interpret this evidence as fully consistent with the pass-through of financial and macroeconomic uncertainty to real economic activity hypothesized in our baseline model. In particular, the data seem to support the fact that in the Great Moderation and Great Recession+Slow Recovery periods financial uncertainty shocks foster greater uncertainty about future economic growth.

These findings on reverse causality allow us to make direct contact with Ludvigson et al. (2018a), the closest paper to ours in this respect. In line with their results, our analysis is consistent with the view that financial uncertainty is a driver of the business cycle, not a reaction to it. According to our identification scheme, however, financial uncertainty affects the business cycle indirectly by triggering greater macroeconomic uncertainty on-impact. Instead we find remarkable differences with Ludvigson et al. (2018a) when we look at the behavior of macroeconomic uncertainty: while they report that macroeconomic uncertainty shocks could be characterized as an endogenous response to business cycle fluctuations and have positive effects on real activity, we find that macroeconomic uncertainty is exogenous to the business cycle and has a negative on-impact effect on real activity. Ludvigson et al. (2018a) base their conclusions on a novel methodology which combines the external instruments approach with the mechanics of set-identification (see also Ludvigson et al., 2018b). The endogeneity of macroeconomic uncertainty they document might reflect the 'asymmetric' characterization of financial and macroeconomic uncertainty shocks implicit in their approach, i.e. the fact that the 'event constraints' are imposed on financial uncertainty only, and that the 'correlation constraints' employ aggregate stock market returns as the only external variable with informational content about uncertainty shocks. Our analysis unveils important time-variation (regime-dependency) in the dynamic responses to uncertainty shocks which could further explain the differences between of restrictions being tested. In the robustness section of the Technical Supplement, we show that all p-values associated with the LR tests discussed in this section increase dramatically once we replace, ceteris paribus, the measure of macroeconomic uncertainty, $U_{M t}$, with an alternative one obtained by 'purging' $U_{M t}$ from a subset of financial variables, denoted $U_{M t}^{p}$. Our choice of using $U_{M t}$ and not directly $U_{M t}^{p}$ in the estimation of our baseline SVAR is motivated by the idea of using the same information set as Ludvigson et al. (2018a) to facilitate comparison. 
their results and ours.

Our empirical evidence fully lines up with Carriero et al. (2018b) as concerns the exogeneity of macroeconomic uncertainty. Carriero et al. (2018b) identify the shocks by a novel stochastic volatility approach based on non-recursive SVARs which include measures of macroeconomic and financial uncertainty, one at a time. In their model the on-impact coefficients are constant but an independent stochastic process drives the volatility of the system and facilitates the identification of the shocks compared to our regime-dependent method. The extension of their approach to the case of three-equations systems is computationally demanding, and this fact probably explains why they do not separately identify the effects of macroeconomic and financial sources of uncertainty on economic fluctuations, and why in their analysis financial uncertainty is not 'as exogenous' as we find in our setup.

IRFs. The implied IRFs are computed as in eq. (15) by replacing $A_{1}, A_{2}$ and $A_{3}$ and $\tilde{B}, \tilde{B}+\tilde{Q}_{2}$ and $\tilde{B}+\tilde{Q}_{2}+\tilde{Q}_{3}$ with their estimates, and are plotted in Figures $2-5$ over an horizon of $h_{\max }=60$ periods ( 5 years). Figure 2 plots the IRFs obtained on the three volatility regimes for $f=1$ (one-month uncertainty). Figures 3-5 plot the IRFs separately for each regime, disentangling the case $f=1$ (one-month uncertainty) from the case $f=12$ (one-year uncertainty) ${ }^{17}$ All plots show responses to one standard deviation changes in $e_{j t}, j=M, Y, F$ in the direction that leads to an increase in its own variable $X_{i t}, i=M, Y, F$, where $X_{M t}=U_{M t}$, $X_{Y t}=Y_{t}$ and $X_{F t}=U_{F t}$, respectively. This normalization allows us to directly compare the responses of real economic activity in the three volatility regimes.

In Figure 2 (which can be fully appreciated in color), the blue IRFs refer to the Great Inflation period, the red IRFs to the Great Moderation period and the yellow IRFs to the Great Recession+Slow Recovery period. The first row reports the response of macroeconomic uncertainty to the three structural shocks, the second row reports the response of industrial production, and the third row reports the response of financial uncertainty. In this case, confidence bands have not been reported to ease reading 18 In order to compare results with a benchmark, Figure 2 also plots the IRFs generated by the SVAR identified by the Lanne and Lütkepohl's (2008) method, discussed at the beginning of this section, i.e. the structural model based on the specification in eq. 20 and regime-invariant autoregressive coefficients.

The graphs in Figure 2 suggest four main comments. First, there is evidence of substantial time variation in the impulse responses: the estimated IRFs differ quantitatively and quali-

\footnotetext{
${ }^{17}$ To save space, a detailed comment of the IRFs in Figures 3-5 can be found in the Technical Supplement

${ }^{18}$ Recall that the reduced form analysis in Section 3.2 shows that there are significant differences between all VAR coefficients (autoregressive parameters and covariance matrices) across the three volatility regimes. Accordingly, the three IRFs in each graph of Figure 2 read as transformations of parameters which have been established to be statistically different in their population values.
} 
tatively across the three volatility regimes. Although uncertainty shocks curb industrial production growth in all three macroeconomic regimes, the persistence of the response and the number of periods after which the negative peak is reached vary across regimes. Second, the effects of macroeconomic uncertainty shocks on all variables are larger and more persistent in the Great Recession+Slow Recovery period. In particular, macroeconomic uncertainty seems to have played a sizable role in driving persistently down economic activity during this period. Third, while real economic activity reacts negatively and persistently to uncertainty shocks, uncertainty reacts only mildly to real activity shocks, if anything. Fourth, there exist differences, as expected, between the IRFs estimated with our non-recursive SVAR and the IRFs produced by keeping the structural parameters fixed.

Overall, combined with the reduced form evidence in Section 3.2 , Figures 2-5 provide a positive answer to our second research question: the short-run relationship between uncertainty and real economic activity changes qualitatively and quantitatively across macroeconomic regimes. A researcher who ignores the regime-dependent nature of uncertainty shocks is likely to estimate compounded effects, which hide the different dynamics displayed in the data.

The estimated IRFs can also be framed in a recent debate on the role of uncertainty during the zero lower bound. According to Plante et al. (2018), during the zero lower bound, which roughly coincides with the Great Recession+Slow Recovery period, macroeconomic variables were more responsive to negative shocks hitting the economy because of the inability of the Fed to use conventional instruments to stabilize the economy, inducing a general increase in the uncertainty surrounding future growth. The IRFs in Figures 4 and 5 show that there is indeed a difference in the response of uncertainty to real economic shocks when moving from the Great Moderation to the Great Recession+Slow Recovery period. However, while this effect helps to explain why the correlation between uncertainty and real economic activity increases after the GFC (see the correlations in Table 1), it is not sufficient to claim that uncertainty is an endogenous (causal) response to real economic activity shocks because according to our analysis the response is at most lagged of one period, but is not instantaneous.

Finally, the estimated IRFs also line up with several contributions in the literature which highlight how uncertainty shocks have had larger effects after the GFC. This can be due to large financial frictions, as in Alfaro et al. (2018), Caggiano et al. (2017b), and Gilchrist et al. (2014), or to the presence of the zero lower bound, as in Caggiano et al. (2017a) and Basu and Bundick (2017). They also support theoretical and empirical research that highlights how uncertainty shocks might have time-varying effects which depend on different macroeconomic conditions like, e.g. the level of financial frictions (Alfaro et al., 2018; Gilchrist et al., 2014, Alessandri and Mumtaz, 2014), the stance of the business cycle (Cacciatore and Ravenna, 2016; 
Caggiano et al., 2014), or the stance of monetary policy (Basu and Bundick, 2017, Caggiano et al., 2017a).

\section{Concluding remarks}

This paper has addressed two controversial issues that characterize the empirical literature on uncertainty: whether time-variation in uncertainty should be considered as an exogenous driver of the business cycle or, rather, an endogenous response to it, and whether the real effects of uncertainty shocks have changed over time with the changes in macroeconomic conditions. The two issues have been analyzed simultaneously with a small-scale non-recursive SVAR estimated on U.S. post-WW2 data, by resorting to an 'identification-through-heteroskedasticity' approach which is novel in the literature on uncertainty. Unlike other existing identification approaches, our framework allows us to jointly estimate regime-dependent effects of uncertainty shocks, and is general enough to account for reverse causality, i.e. to allow for a contemporaneous response of both real activity to uncertainty shocks and of uncertainty to real activity shocks.

Empirical results suggest that there are important differences in the impact and propagation mechanism of uncertainty shocks across the three main macroeconomic regimes that characterize the U.S. business cycle, and that uncertainty, both macro and financial, is better approximated as an exogenous source of economic decline rather than an endogenous response to it. We find that macroeconomic uncertainty shocks have always had a contractionary impact on real activity, but that these effects have become larger since the GFC. In turn, after the 1980s, financial uncertainty shocks affect real economic activity by fostering greater macroeconomic uncertainty.

Overall, our findings support the theoretical models where uncertainty is treated as an exogenous driver of economic fluctuations, as in e.g. Bloom (2009) and Basu and Bundick (2017), and the empirical specifications where uncertainty enters recursive SVARs. In this respect, our analysis is partially consistent with the evidence reported in Ludvigson et al. (2018a) and is not at odds with Carriero et al. (2018b).

\section{Acknowledgments}

The authors thank for constructive comments on previous versions of this article the Co-Editor, Michael McCracken, two anonynous referees, Piergiorgio Alessandri, Efrem Castelnuovo, Toru Kitagawa, Haroon Mumtaz, Giovanni Pellegrino, Chiara Scotti, seminar participants at the "Padova Macro Talks 2017", Queen Mary University and the Bank of Italy, and conference participants at the "2018 IAAE Annual Conference", Montreal, June 2018; the "11th International 
Conference of Computational and Financial Econometrics (CFE 2017)" London, December 2017. We are solely responsible for any remaining errors. The second author gratefully acknowledges partial financial support from University of Milan research grant "Linea 2"; the third author gratefully acknowledges financial support from the Australian Research Council via the Discovery Project DP160102281; the fourth author gratefully acknowledges partial financial support from RFO grants from the University of Bologna.

\section{References}

Aastveit, K.A., Carriero, A., Clark, T.D., \& Marcellino, M. (2017). Have standard VARs remained stable since the crisis? Journal of Applied Econometrics 32, 931-951.

Alessandri, P., \& Mumtaz, H. (2018). Financial regimes and uncertainty shocks. Journal of Monetary Economics, forthcoming.

Alfaro, I.N., Bloom, N., \& Lin, X. (2018). The finance-uncertainty multiplier. NBER Working Paper 24571.

Arellano, C., Bai, Y., \& Kehoe, P. (2018). Financial frictions and fluctuations in volatility. Journal of Political Economy, forthcoming.

Bacchiocchi, E., \& Fanelli, L. (2015). Identification in Structural Vector Autoregressive models with structural changes, with an application to U.S. monetary policy. Oxford Bulletin of Economics and Statistics 77, 761-779.

Bacchiocchi, E., Castelnuovo, E., \& Fanelli, L. (2018). Give me a break! Identification and estimation of the macroeconomic effects of monetary policy shocks in the U.S. Macroeconomic Dynamics, forthcoming.

Bachmann, R., \& Moscarini, G. (2012). Busyness cycles and endogenous uncertainty. Unpublished Manuscript, Aachen University.

Basu, S., \& Bundik, B. (2017). Uncertainty shocks in a model of effective demand. Econometrica, forthcoming.

Bloom, N. (2009). The impact of uncertainty shocks. Econometrica 77, 623-685.

Brüggemann, R., Jentsch, C., \& Trenkler, C. (2016). Inference in VARs with conditional volatility of unknown form. Journal of Econometrics 191, 69-85. 
Cacciatore, M., \& Ravenna, F. (2016). Uncertainty, Wages, and the Business Cycle. Unpublished Manuscript.

Caggiano, G., Castelnuovo, E., \& Groshenny, N. (2014). Uncertainty shocks and employment dynamics in U.S. recessions. Journal of Monetary Economics 67, 78-92.

Caggiano, G., Castelnuovo, E., \& Pellegrino, G. (2017a). Estimating the Real Effects of Uncertainty Shocks at the Zero Lower Bound. European Economic Review 100, 257-272.

Caggiano, G., Castelnuovo, E., Delrio, S., \& Robinson, T. (2017b). Time-dependent financeuncertainty multipliers. Working Paper.

Caldara, D., Fuentes-Albero, C., Gilchrist, S., \& Zakrajšek, E. (2016). The macroeconomic impact of financial and uncertainty shocks. European Economic Review 88, 185-207.

Caldara, D., \& Scotti, C. (2018). Uncertainty and financial stability. Paper presented at the 2018 IAAE Annual Conference of the International Association for Applied Econometrics.

Carriero, A., Mumtaz, H., \& Theodoridis, K. (2015). The impact of uncertainty shocks under measurement error: A proxy SVAR approach. Journal of Money, Credit, and Banking 47, 1223-1238.

Carriero, A., Clark, T.E., \& Marcellino, M. (2018a). Measuring uncertainty and its impcat on the economy. Review of Economics and Statistics, forthcoming.

Carriero, A., Clark, T.E., \& Marcellino, M. (2018b). Endogenous uncertainty. Federal Reserve Bank of Cleveland, Working Paper 18/05.

Chen, W., \& Netšunajev, A. (2018). Structural Vector Autoregressions with time varying transition probabilities. Bank of Estonia, Working Paper Series 2/2018

Christriano, J.C., Motto, R., \& Rostagno, M. (2014). Risk shocks. American Economic Review 104, 27-65.

Cúrdia, V., Del Negro, M., \& Greenwald, D.L. (2014). Rare shocks, great recession. Journal of Applied Econometrics 29, 1031-1052.

Fajgelbaum, P., Taschereau-Dumouchel, M., \& Schaal, E. (2016). Uncertainty traps. Quarterly Journal of Economics 132, 1641-1692.

Forni, M., \& Gambetti, L. (2014). Sufficient information in structural VARs. Journal of Monetary Economics 66, 124-136. 
Gilchrist, S., Sim, J., \& Zakrajsek, E. (2014). Uncertainty, financial frictions and investment dynamics. NBER Working Paper No. 20038.

Gourio, F. (2014). Financial distress and endogenous uncertainty. Working Paper, Federal Reserve Bank of Chicago.

Jurado, K., Ludvigson, S.C., \& Ng, S. (2015). Measuring uncertainty. American Economic Review 105(3), 1177-1216.

Kilian, L. (1998). Small-sample confidence intervals for impulse response functions. Review of Economics and Statistics 80, 218-230.

Kilian, L., \& Lütkepohl, H. (2017). Structural Vector Autoregressive Analysis. Cambridge University Press, Cambridge.

Lanne, M., \& Lütkepohl, H. (2008). Identifying monetary policy shocks via changes in volatility. Journal of Money, Credit and Banking 40, 1131-1149.

Lanne, M., Lütkepohl, H., \& Maciejowska, K. (2010). Structural vector autoregressions with Markov switching. Journal of Economic Dynamics and Control 34, 121-131.

Lewis, D.J. (2018). Identifying shocks via time-varying volatility. Working Paper, Harvard University, version $03 / 14 / 2018$.

Ludvigson, S.C., Ma, S., \& Ng, S. (2018a). Uncertainty and business cycles: exogenous impulse or endogenous response? Working Paper, draft dated May 9, 2018.

Ludvigson, S.C., Ma, S., \& Ng, S. (2018b). Shock restricted Structural Vector-Autoregressions. Working Paper, draft dated July 24, 2018.

Lütkepohl, H. (2013). Vector Autoregressive Models. in N. Hashimzade and M.A. Thornton (Eds.), Handbook of Research Methods and Applications in Empirical Macroeconomics, Cheltenham: Edward Elgar, 139-164.

Lütkepohl, H., \& Netšunajev, A. (2017). Structural vector autoregressions with heteroskedasticity: A review of different volatility models. Econometrics and Statistics 1, 2-18.

Magnusson, L. M., \& Mavroeidis, S. (2014). Identification using stability restrictions. Econometrica 82, 1799-1851.

McConnell, M.M., \& Perez-Quiros, G. (2000). Output fluctuations in the United States: what has changed since the early 1980's? American Economic Review 90, 1464-1476. 
McCracken, M.W., and Ng, S. (2015). FRED-MD: A monthly database for macroeconomic research. Journal of Business and Economic Statistics 34, 574-589.

Mertens, K., and Ravn, M. (2013). The dynamic effects of personal and corporate income tax changes in the United States. American Economic Review 103, 1212-1247.

Mumatz, H., and Theodoridis, K. (2018). The changing transmission of uncertainty shocks in the US: An empirical analysis. Journal of Business and Economic Statistics, forthcoming.

Navarro, G. (2014). Financial crises and endogenous volatility. Working Paper, New York University.

Ng, S., \& Wright, J.H. (2013). Facts and challenges from the Great Recession for forecasting and macroeconomic modeling. Journal of Economic Literature 51, 1120-1154.

Plante, M., Richter, A.W., \& Throckmorton, N.A. (2018). The Zero Lower bound and endogenous uncertainty. The Economic Journal, forthcoming.

Podstawski, M., \& Velinov, A. (2018). The state dependent impact of bank exposure on sovereign risk. Journal of Banking and Finance 88, 63-75.

Rigobon, R. (2003). Identification through heteroskedasticity. Review of Economics and Statistics $85,777-792$.

Rigobon, R., \& Sack B. (2003). Measuring the reaction of monetary policy to the stock market. Quarterly Journal of Economics 118, 639-669.

Rossi, B., Sekhposyan, T., \& Soupre, M. (2016). Understanding the sources of macroeconomic uncertainty. CEPR Discussion Paper No. DP11415.

Scotti, C. (2016). Surprise and uncertainty indexes: Real-Time aggregation of real-activity macro surprises. Journal of Monetary Economics 82, 1-19.

Stock, J.H., \& Watson, M.W. (2012). Disentangling the channels of the 2007-2009 recession. Brooking Panel of Economic Activity, Conference Paper March 22-23.

Stock, J.H., \& Watson, M.W. (2018). Identification and estimation of dynamic causal effects in macroeconomics using external instruments. The Economic Journal 128, 917-948.

Van Nieuwerburgh, S., \& Veldkamp, L. (2006). Learning asymmetries in real business cycles. Journal of Monetary Economics 53, 753-772. 

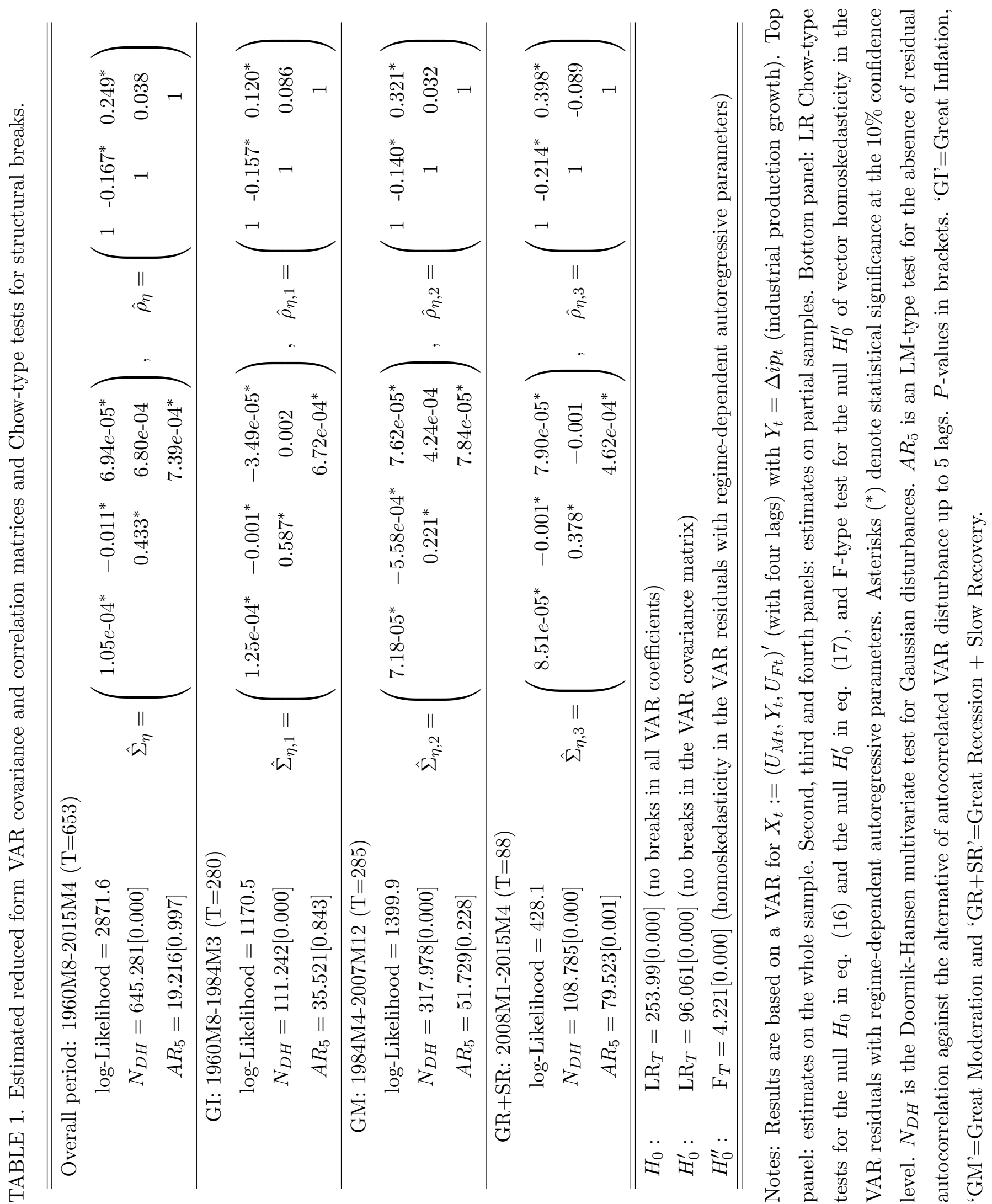


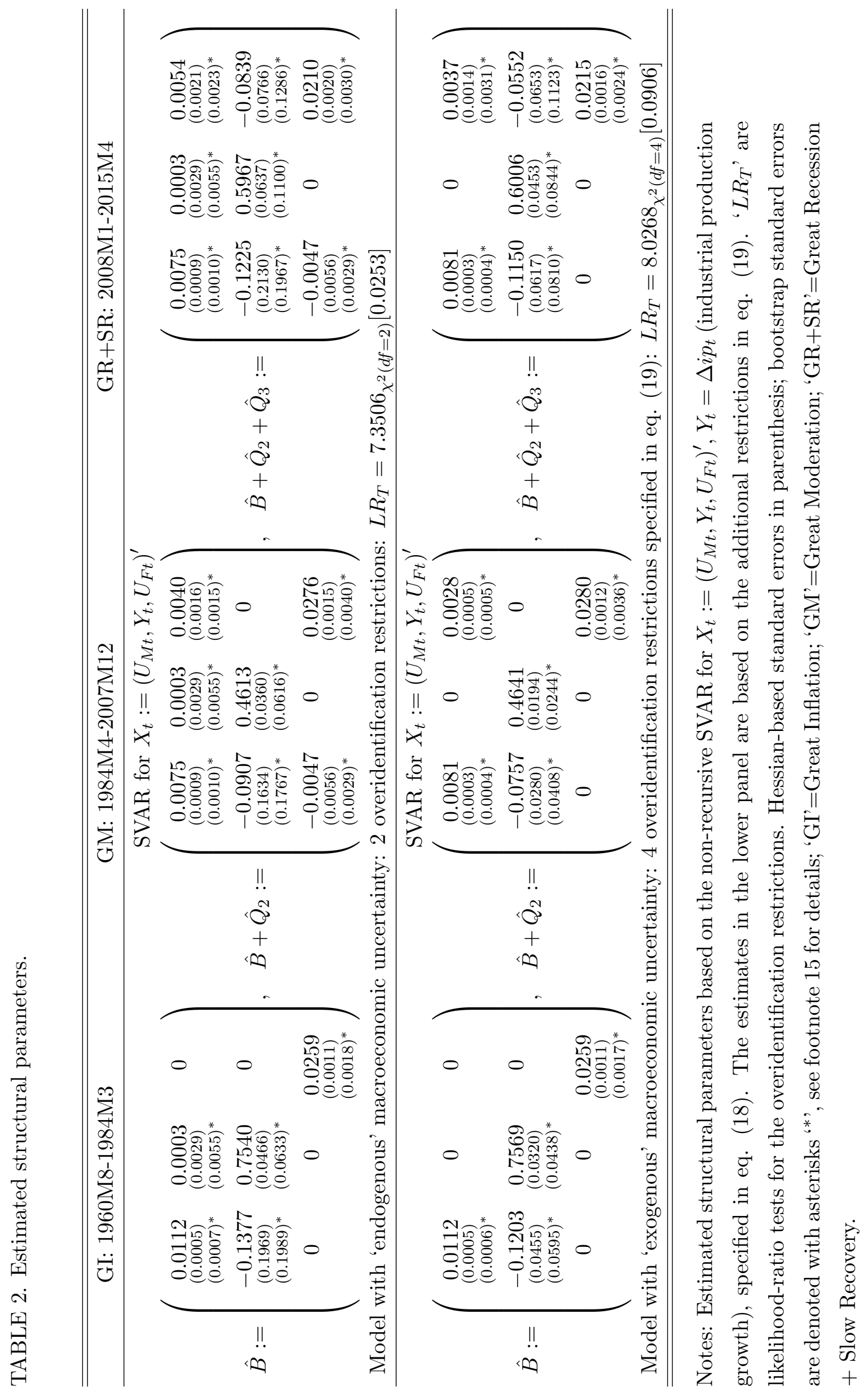



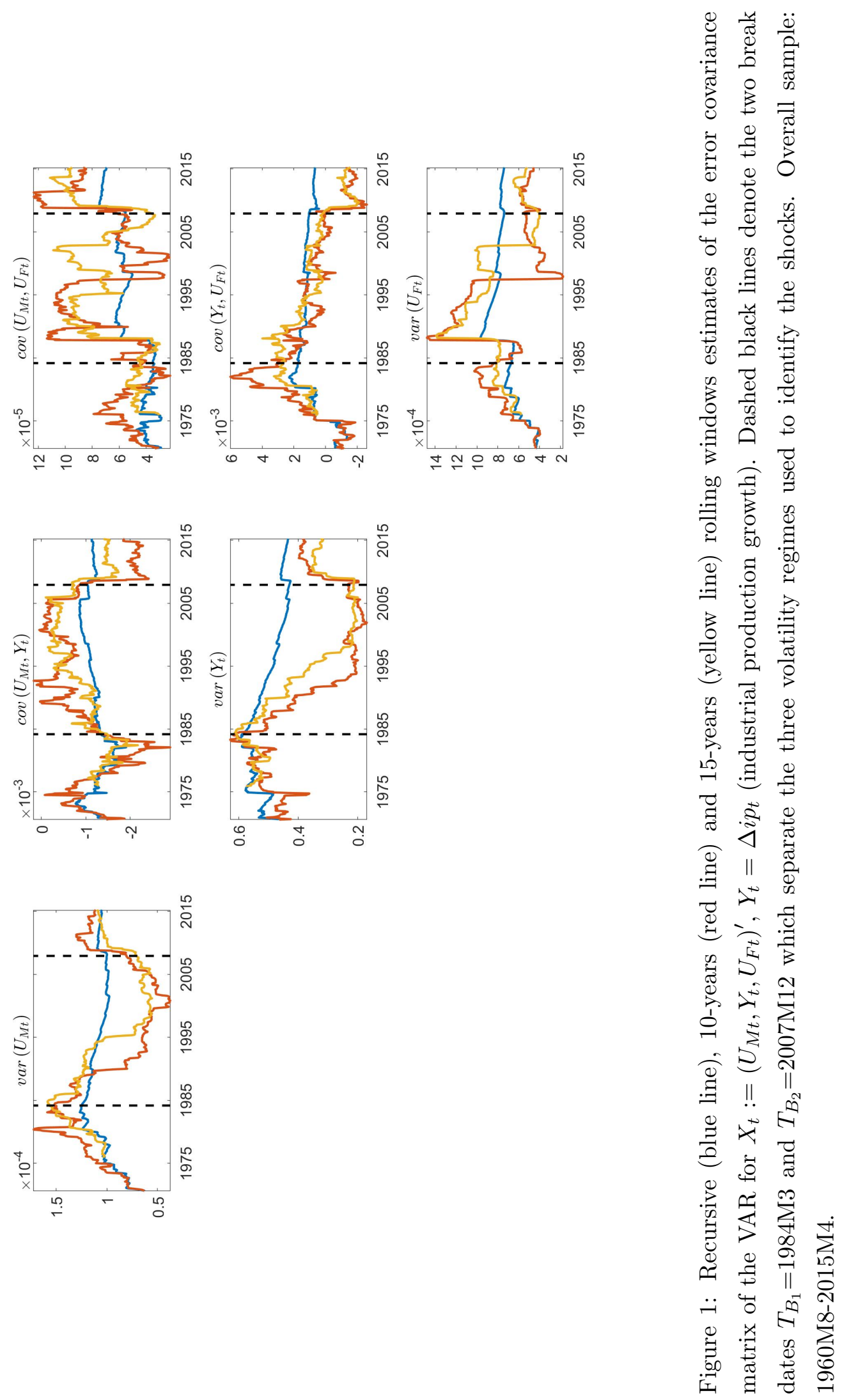

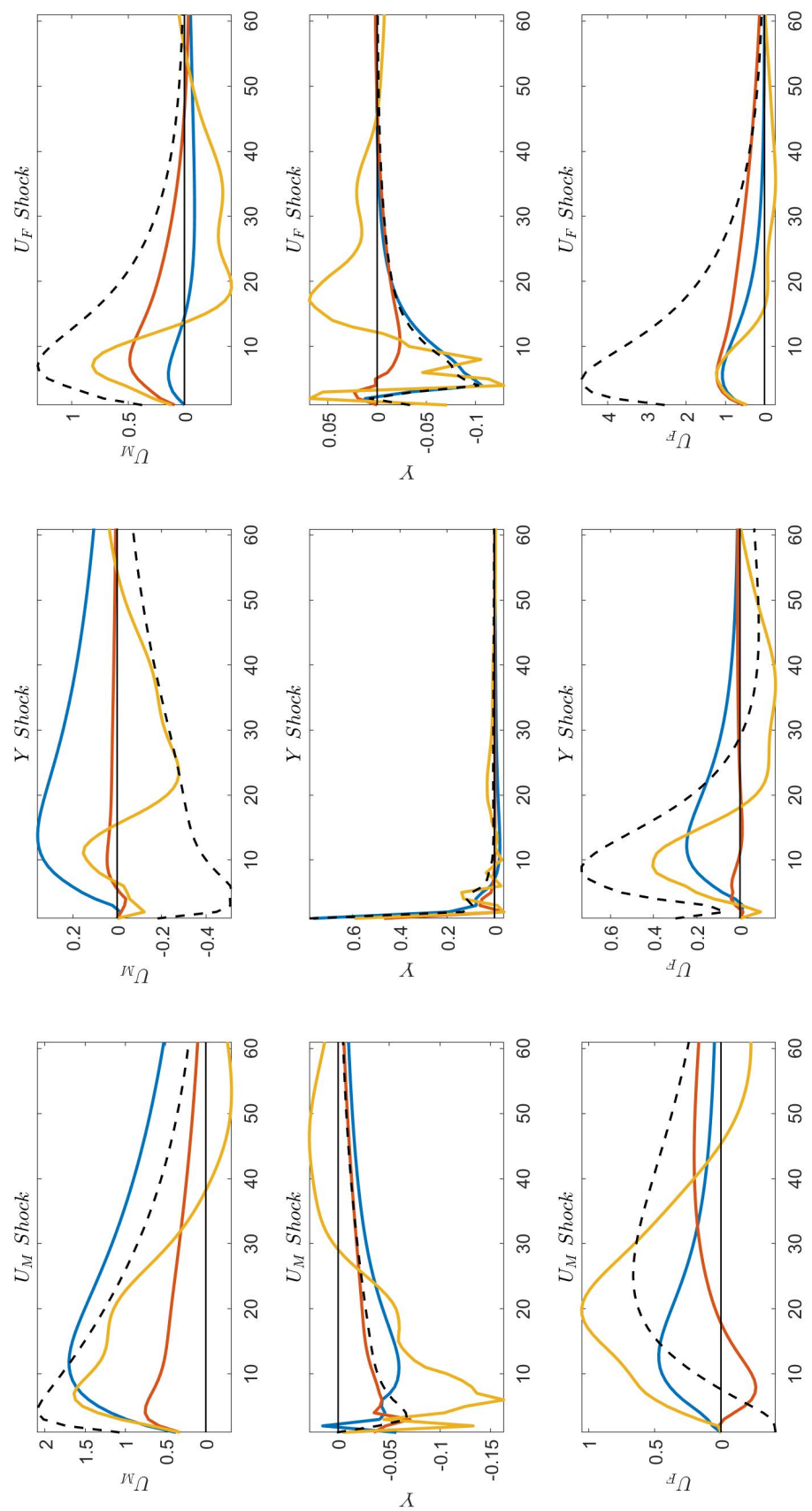

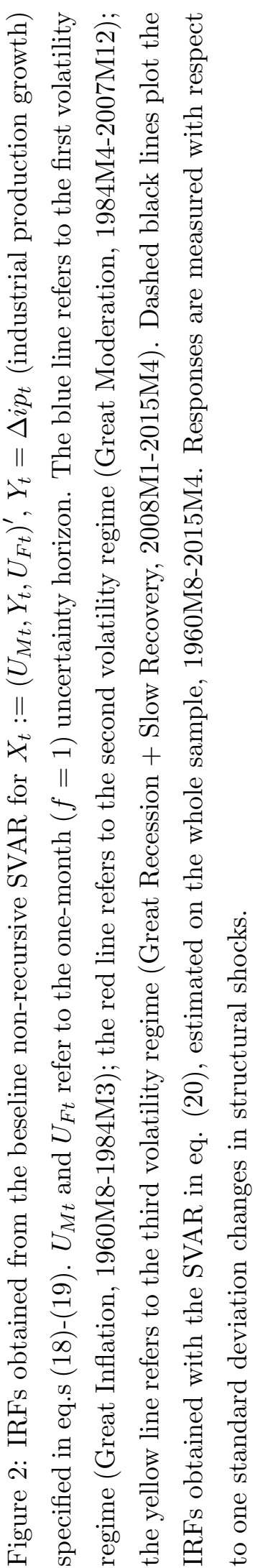



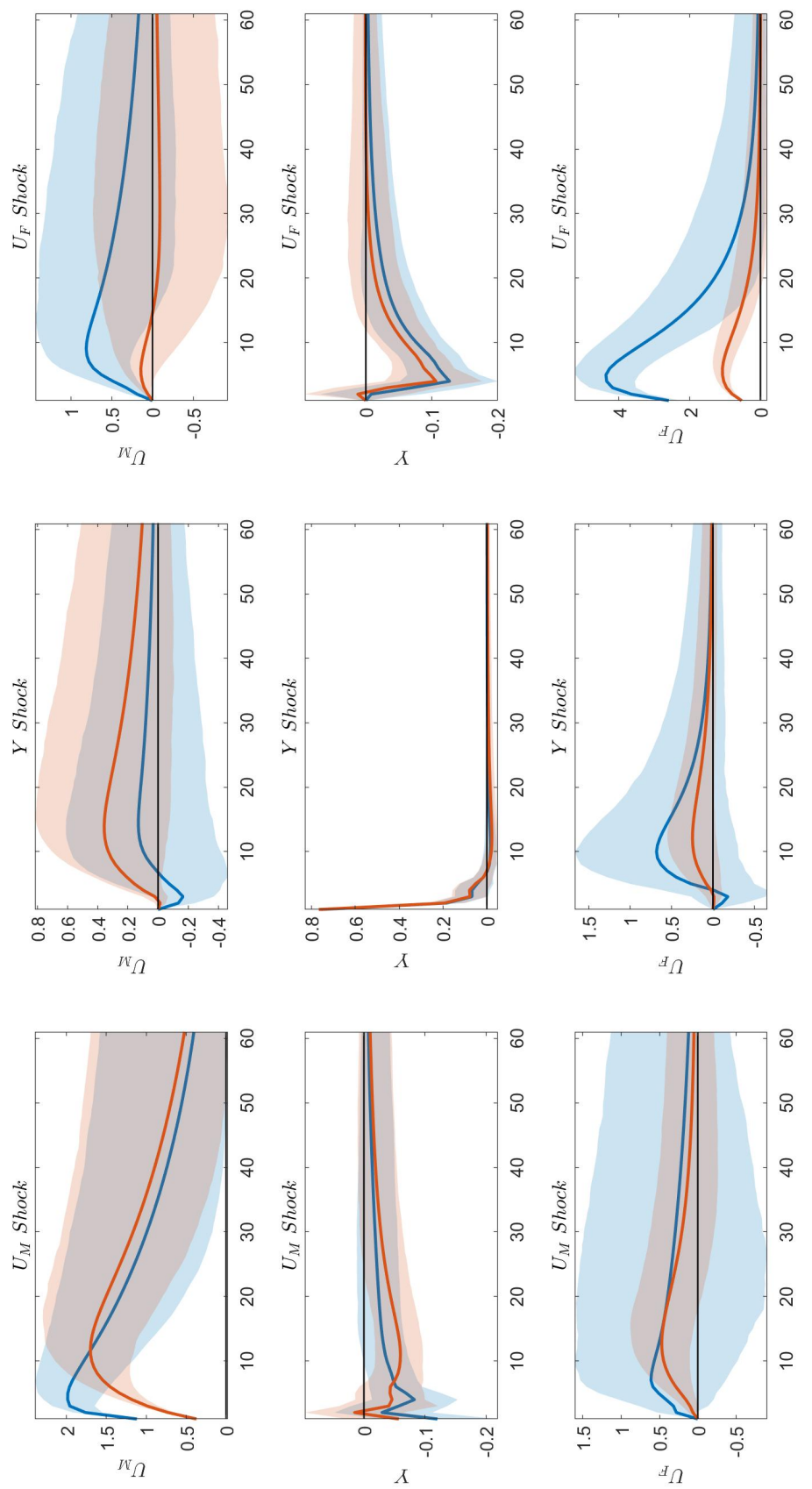

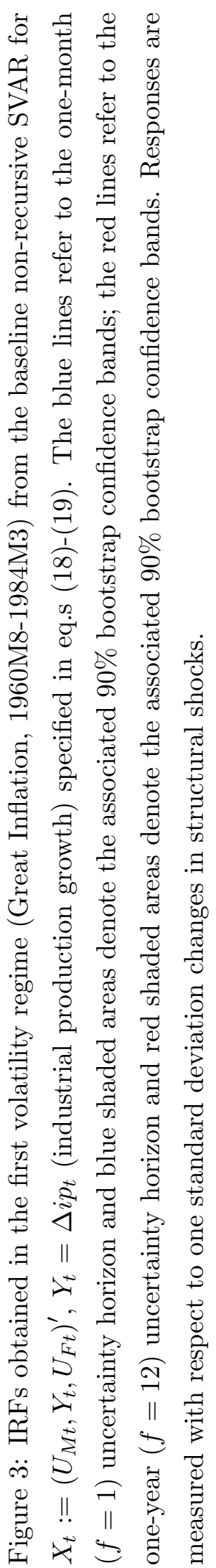



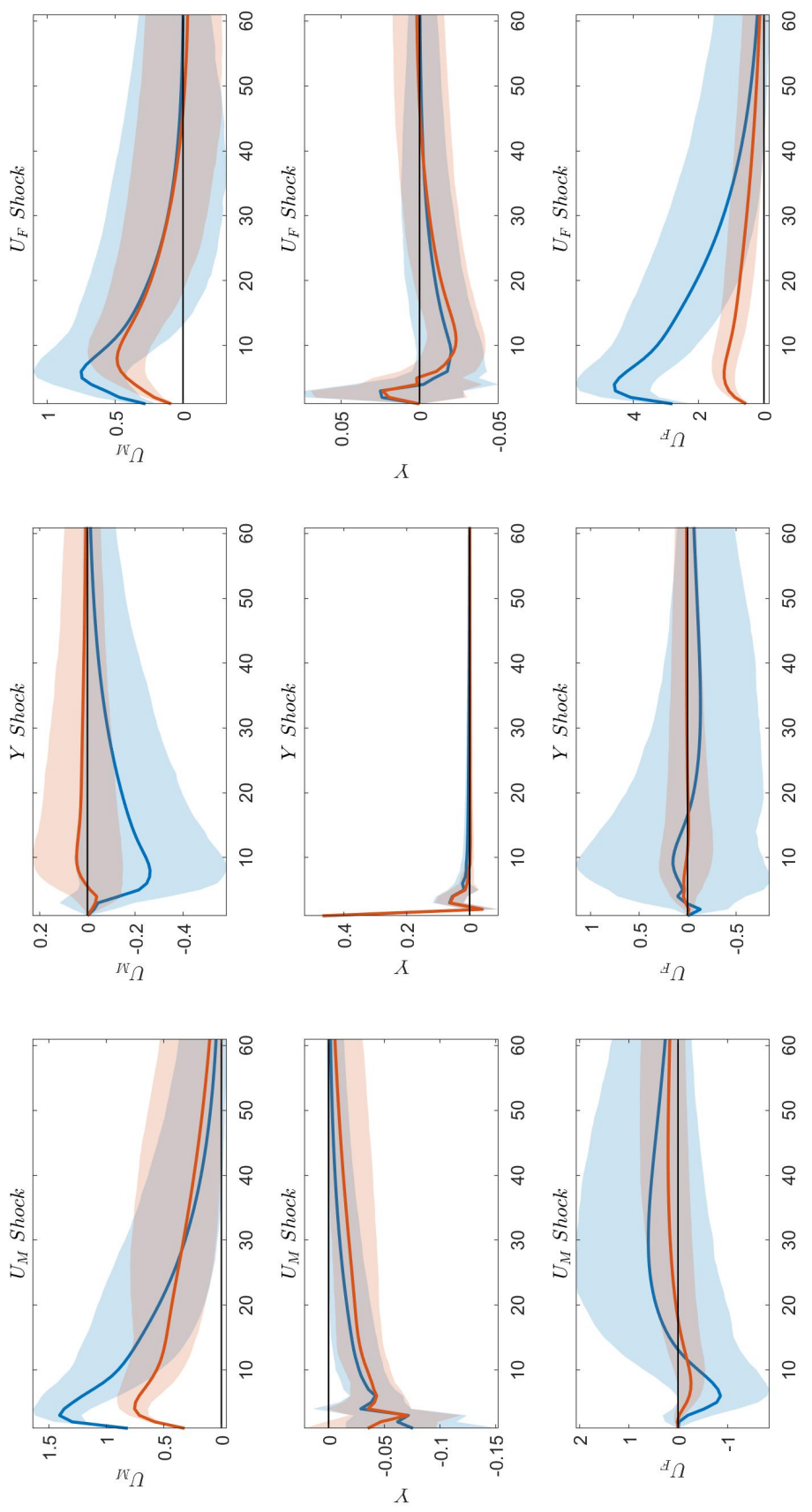

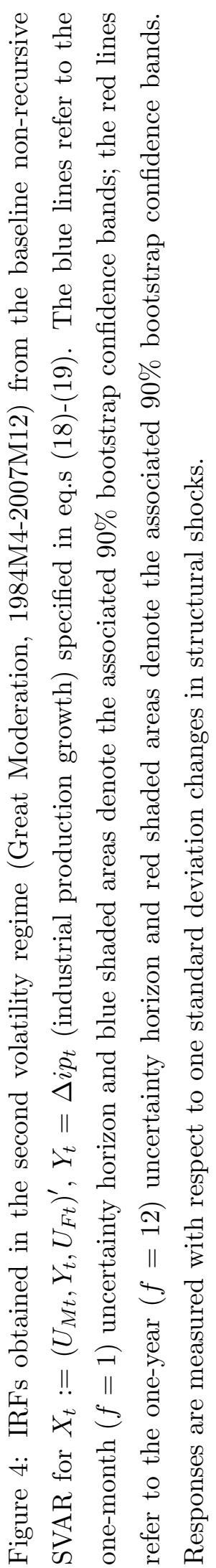



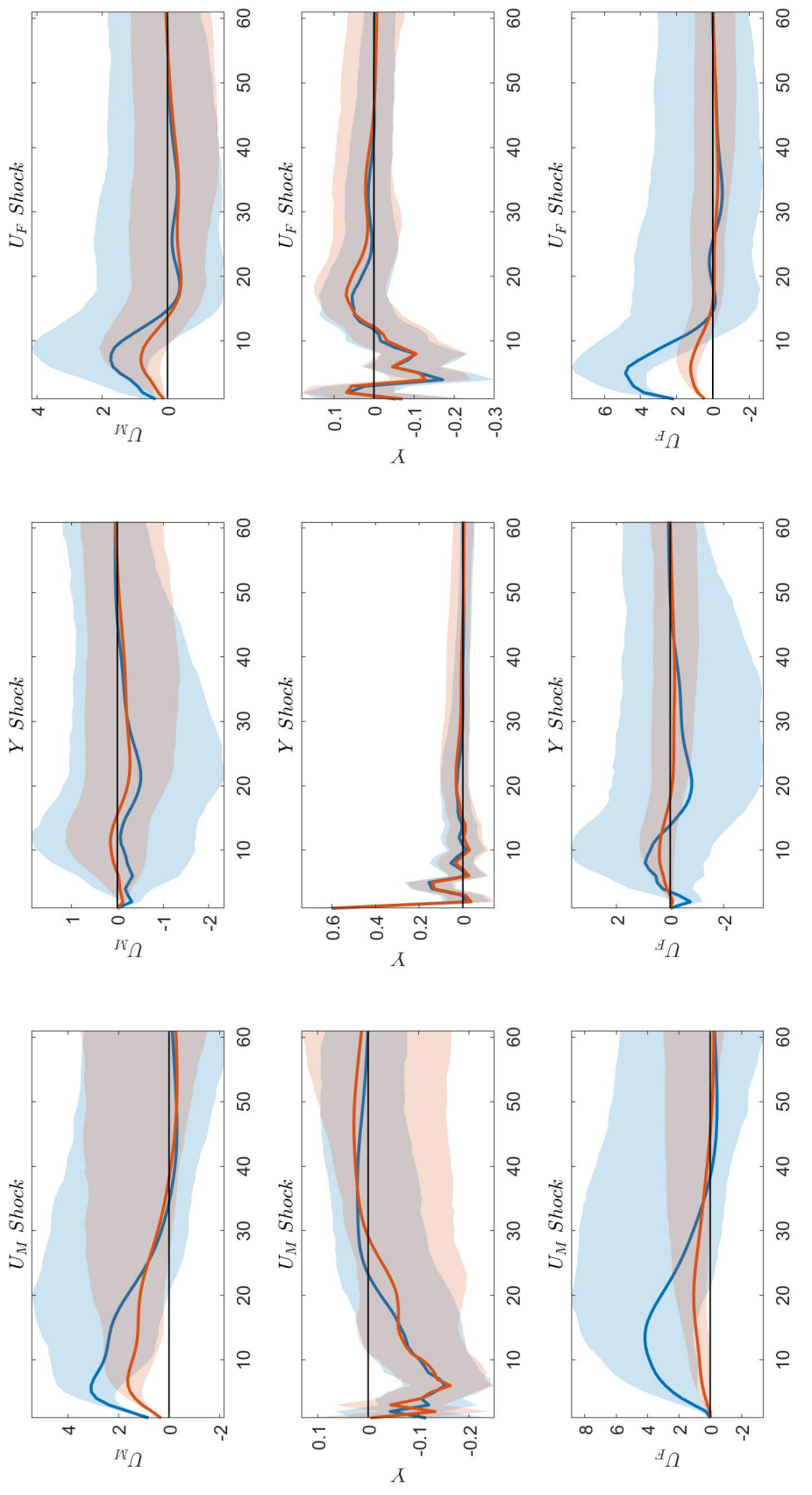

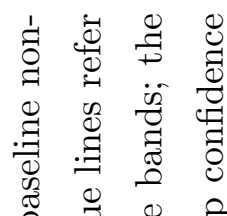

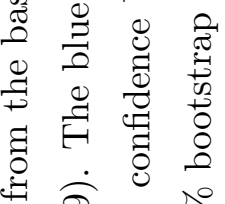

Æ

点我苟 荡

㲾

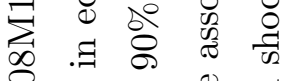

尺

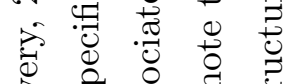

की

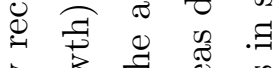

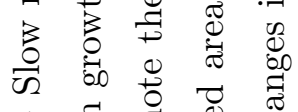

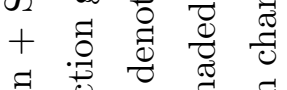

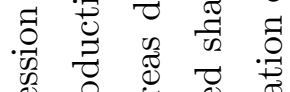

ठ্․

थ

芯

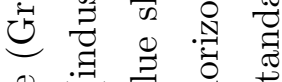

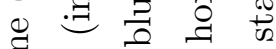

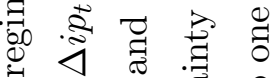

: $\|$ :

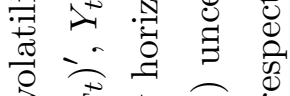

$>$ ए

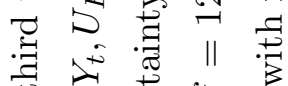

0 过

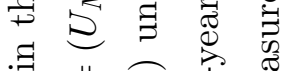

च !

茮芒

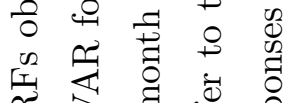

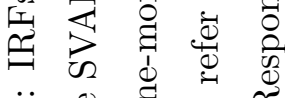

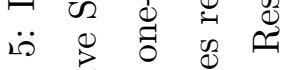

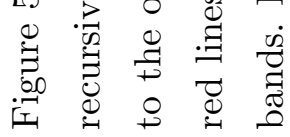

